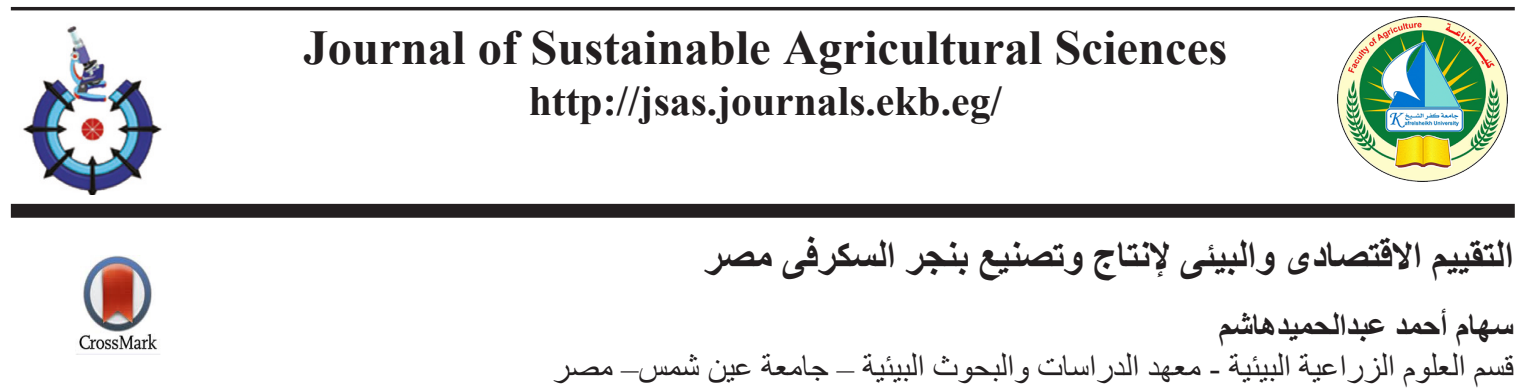

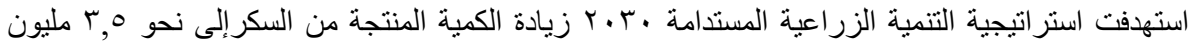

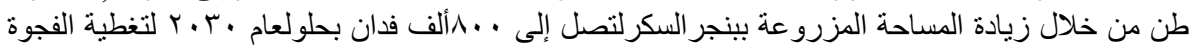

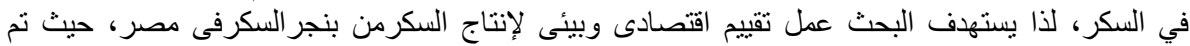

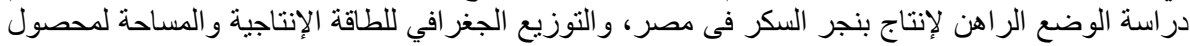

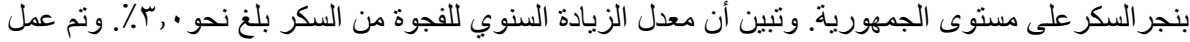

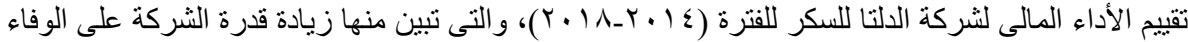

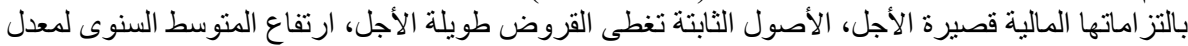

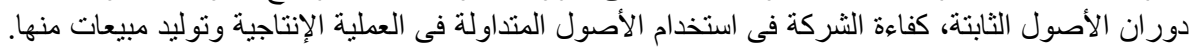

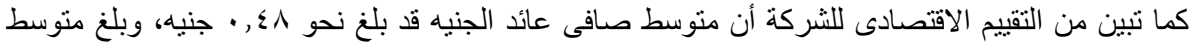

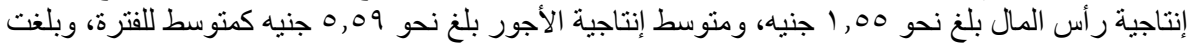

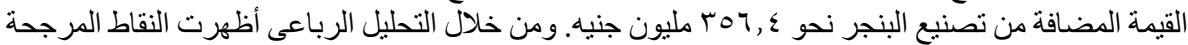

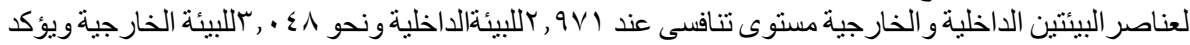
ارتفاع النقاط المرجحة للبيئة الخارجية على النقاط النقاجية المرجحة للبيئة الداخلية إمكانية الاستفادة من عناصر البيئة الخارجية وإمكانية/لتوسع فى إنتاج السكر من بنجر السكر. الكلمات الدالة: بنجر السكر، الاكتفاء الذاتى من السكر، الأداء المالى، التقييم الاقتصادى، نموذج البيانات المقطعية، مصنع الدلتا للسكر.

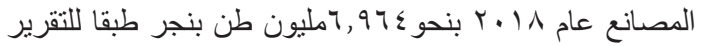

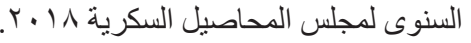

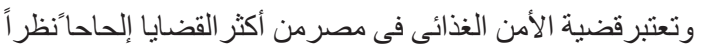

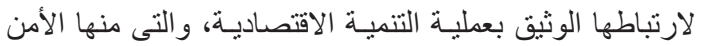

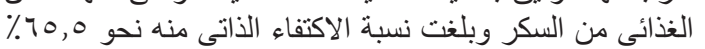

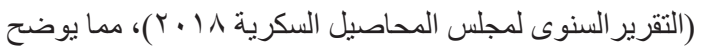

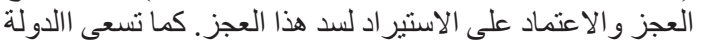

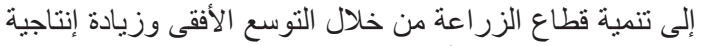

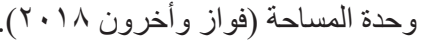

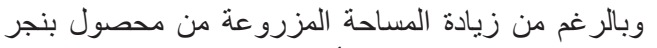

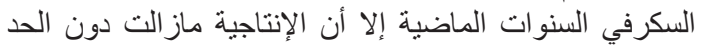

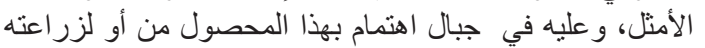

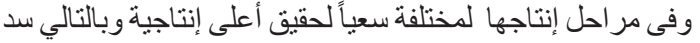

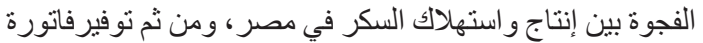
الاستير اد من الخارج (الجزار و أخرون 9 ( • ب).

مشكلة البحث

مرت مصر بمرحلة اكتفاء ذاتى فى أو ائل السبعينات حيث

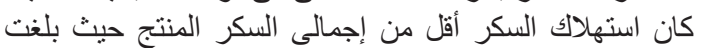

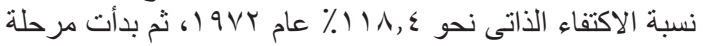

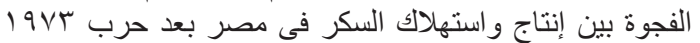

المقدمة

تعتبر صناعة السكر عالمياً من الصناعات التحويلية الهامة والتى التى التئي

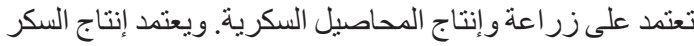

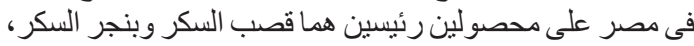

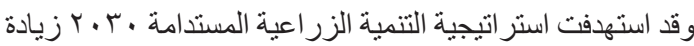

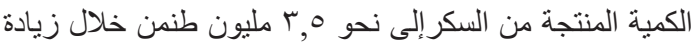

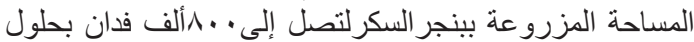

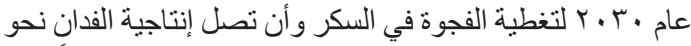

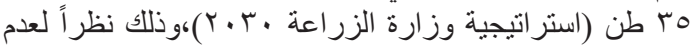

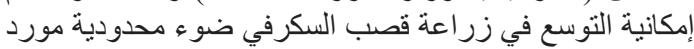

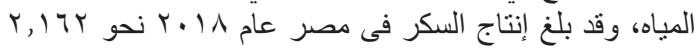

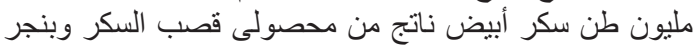

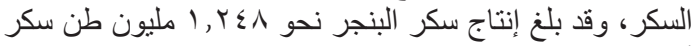

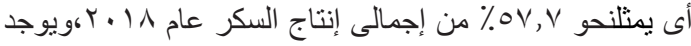

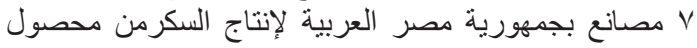

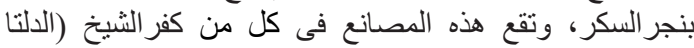

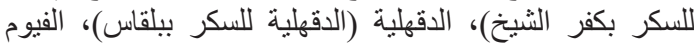

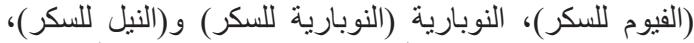

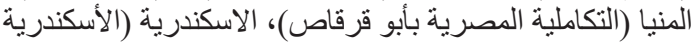

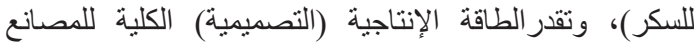

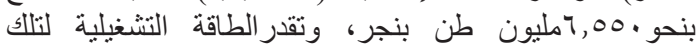




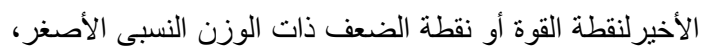

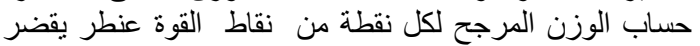

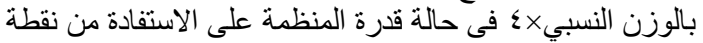

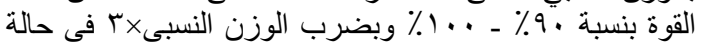

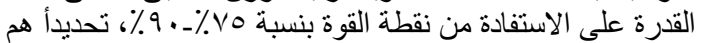

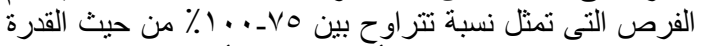

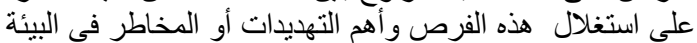

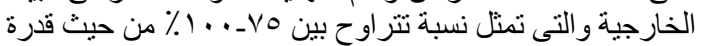

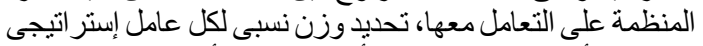

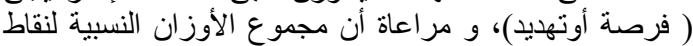

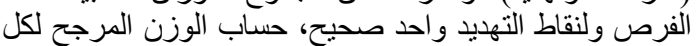

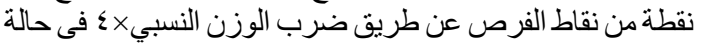

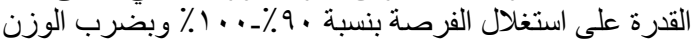

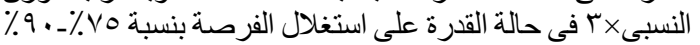

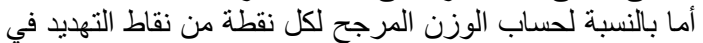

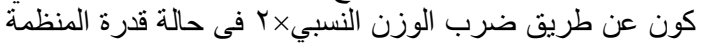

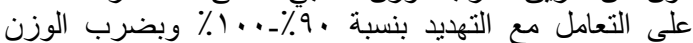

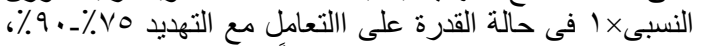
و ونه ينم تحديد انسب الاستر اتيجات وفقاً لمصفو فلة العو امل الداخلية والخارجية.

مصادر البيانات

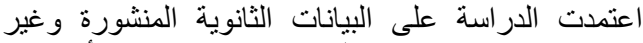

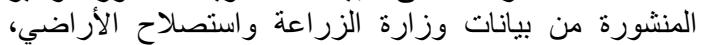

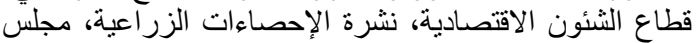

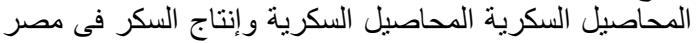

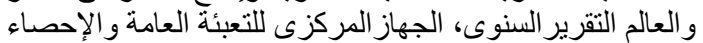

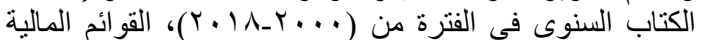

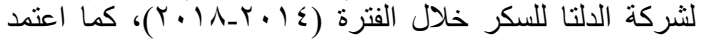

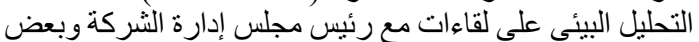

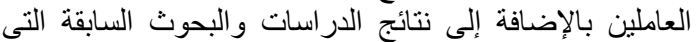

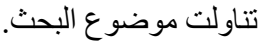
النتائتج البحثية ومناقشتها

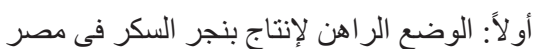

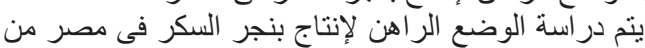
خلال بعض المؤشرات الإنتاجية والإقتصادية لمناجية لمحصول بنجر

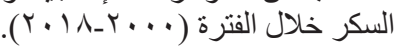

اـ تطور المساحة المزروعة من بنجر السكر:

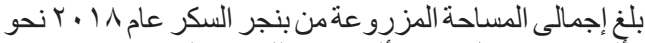

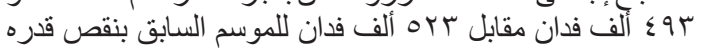

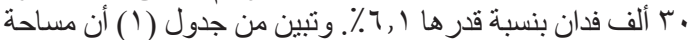

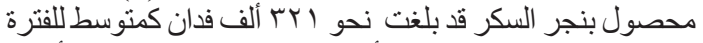

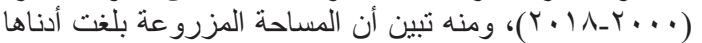

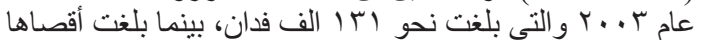

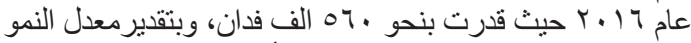

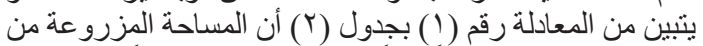

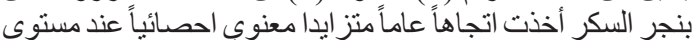

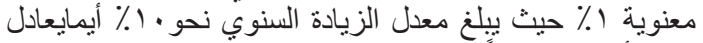

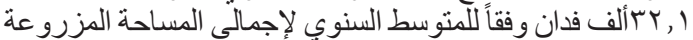
من بنجر السكر خلال فترة الار اسة.

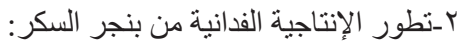

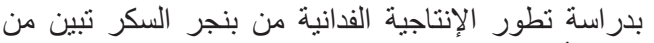

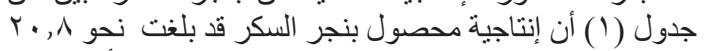

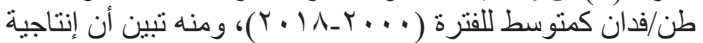

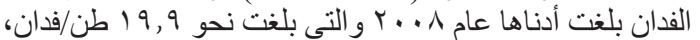

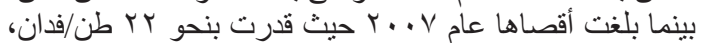

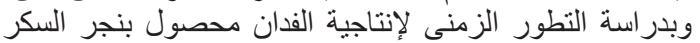

ومع الانفتاح الاقتصادى وما تبعه من زيادة في دخول فئات عديدة

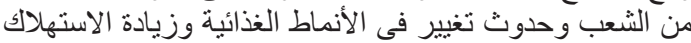

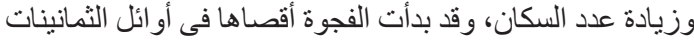

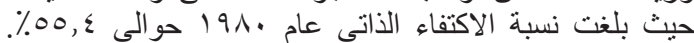

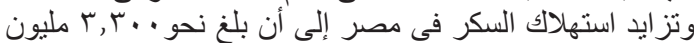

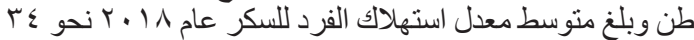

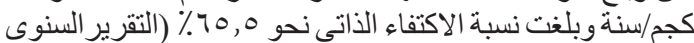

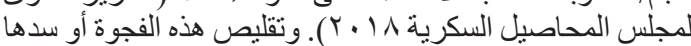

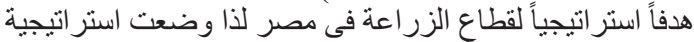

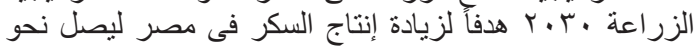

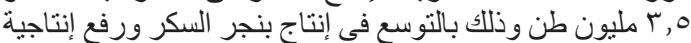

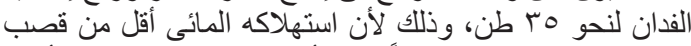

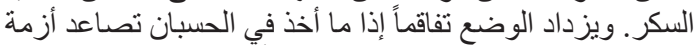

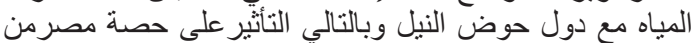

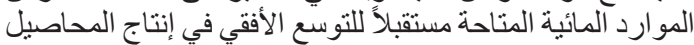
الحقلية ومنها المحاصيل السكرية.

هدف البحث

يستهدف البحث بصفة رئيسية عمل تقييم اقتصادى وبيئى

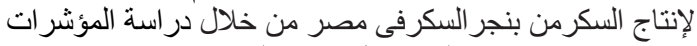

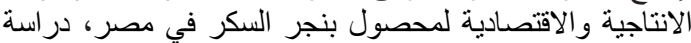

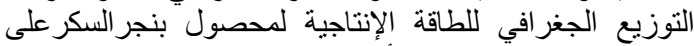

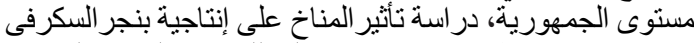

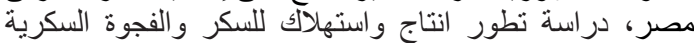

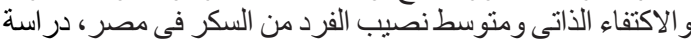

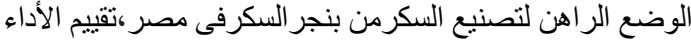

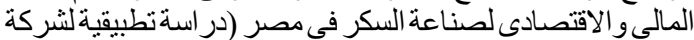
الدلتاللسكر )، التحليل البيئى لإنتاج وصناعناعة السكر من بنجر السكر.

الطريقة البحثية

اعتمد البحث في تحقيق أهدافه على أساليب التحليل الإحصائي

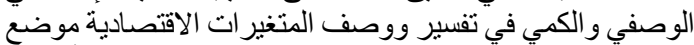

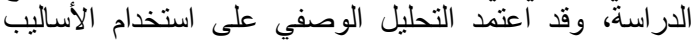

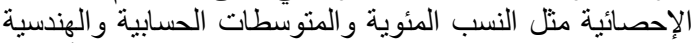

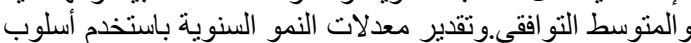

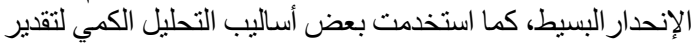

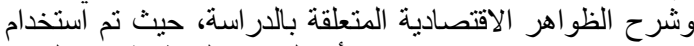

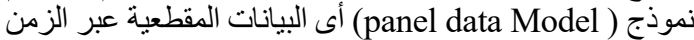

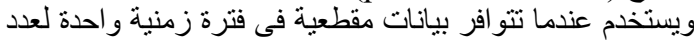

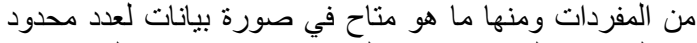

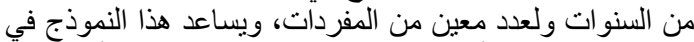

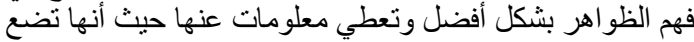

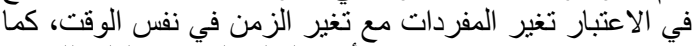

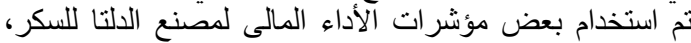

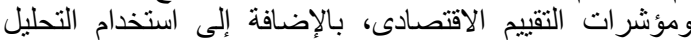

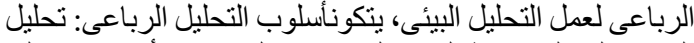

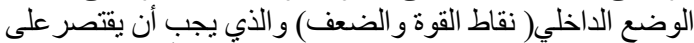

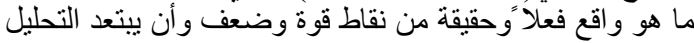

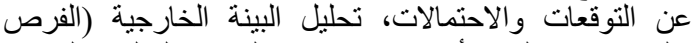
و التهديدات) و الذي يأخذ بعين الاعتبار الوضع الوضينة الفعلي والحقية ولفيقي

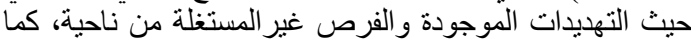

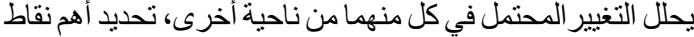

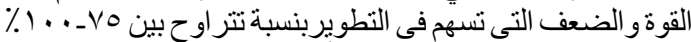

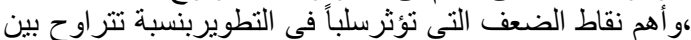

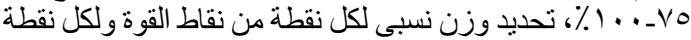

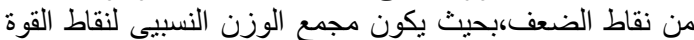
ونقاط الضعف واحد صحيح، ترنيب العو امل الاستر اتيجية الداخلية التونية

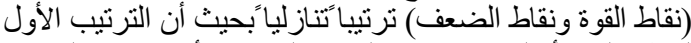

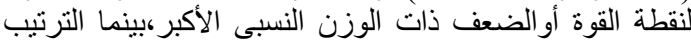

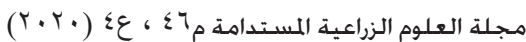




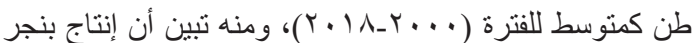

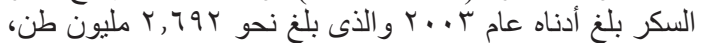

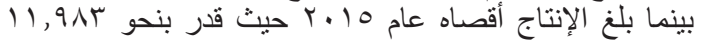

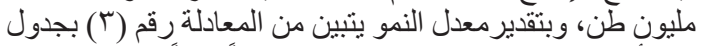

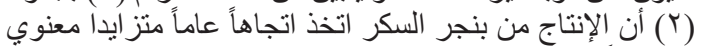

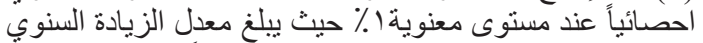

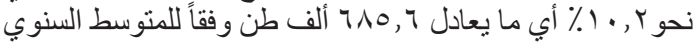
لإجمالي إنتاج بنجر السكر خلادل فترة الدراسة.

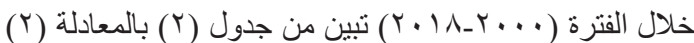

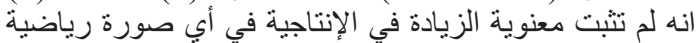

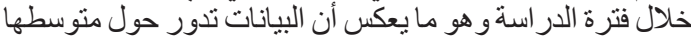
(ثبات نسبي).

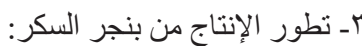

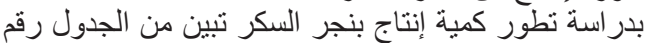

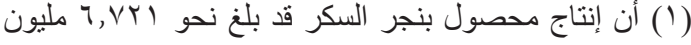

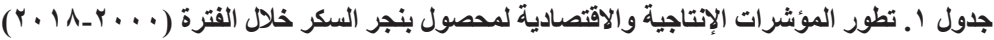

\begin{tabular}{|c|c|c|c|c|c|c|c|c|c|}
\hline المستثمر جنيه/فذاند الجنيه & نتكبة الإيراد إلى & بالجنئ & إجنيالى العائد & جنية/فلنانة الكاليف & بالجنيه/ طنرعي السعر & 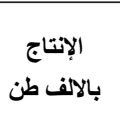 & طن الإنتاجية & فالالف & السنوات \\
\hline$\cdot, 0$ & 1,0 & VII & r $10 \varepsilon$ & $1 \leqslant T r$ & $1 \ldots$ & ห^q. & $r 1, r$ & 154 & $r \ldots$ \\
\hline$\cdot$ • r & $1, r$ & $\varepsilon \wedge$. & $r \ldots$ & lor. & $1 \ldots$ & rAOA & $r_{\bullet}, \cdot$ & $1 \leqslant r$ & $r \ldots l$ \\
\hline$\cdot, 0$ & 1,0 & VIr & rrv. & 1001 & 11. & T171 & $r \cdot, \tau$ & $10 \leqslant$ & $r . . r$ \\
\hline •, & $r, 1$ & 091 & or. 1 & 1770 & 11. & rדवY & $r_{\cdot}, 0$ & 14 & $r \ldots r$ \\
\hline$\cdot, \wedge$ & $1, v$ & $1 \leqslant r r$ & TYYY & INOV & 101 & YATा & $r \cdot r$ & $1 \leqslant 1$ & $r \ldots \varepsilon$ \\
\hline$\cdot, 9$ & 1,9 & 1100 & r411 & 1107 & 17. & $r \leqslant r$. & $r \cdot, 0$ & 178 & r... \\
\hline$\cdot, 9$ & 1,9 & IVTY & $r 4.1$ & 1414 & $|v|$ & $r q .0$ & $r \cdot, q$ & 114 & $r \ldots r$ \\
\hline $1, r$ & $r, r$ & $r \leqslant \wedge q$ & 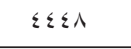 & 1909 & 111 & $0 \leqslant 01$ & $r, \cdot$. & $r \leqslant \wedge$ & $r \ldots r$ \\
\hline 1,1 & $r, 1$ & rove & ¿१ะ१ & rrTh & TrI & TrT & 19,9 & rol & $r \ldots \lambda$ \\
\hline 1,7 & $r, T$ & \&rr. & TAYV & r79V & TIV & צזים & $r \cdot, r$ & ryo & $r \ldots q$ \\
\hline $1, \cdot$ & $r, \cdot$ & $r .01$ & $7.0 \xi$ & $r \ldots r$ & אדיץ & $V \wedge \varepsilon$. & $r \cdot, r$ & TAT & $r .1$. \\
\hline $1, \varepsilon$ & $r, \varepsilon$ & EVYq & 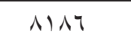 & $r \leqslant 0 V$ & roo & $V \leqslant \wedge \uparrow$ & $r \cdot, V$ & זדו & $r .11$ \\
\hline 1,1 & $r, 1$ & $\sum T Y \wedge$ & AVY. & $\varepsilon .9 r$ & T4 & $91 Y 4$ & $r 1,0$ & $\varepsilon r \varepsilon$ & $r \cdot I r$ \\
\hline 1,1 & $r, 1$ & $\sum 909$ & 9ror & \& & TAV & $1 \ldots \leq \varepsilon$ & $r 1, \Lambda$ & $\leq 7$. & $r .1 T$ \\
\hline$\cdot, 9$ & 1,9 & $\varepsilon \mid v$. & 9.49 & $\{\wedge 79$ & rV. & $11 \cdot \leq 7$ & $r_{1}, 9$ & $0 . \varepsilon$ & $r \cdot 1 \varepsilon$ \\
\hline$\cdot, V$ & $1, v$ & TATA & $910 \xi$ & OrIt & rVq & $119 \Delta r$ & $r 1, r$ & 000 & $r .10$ \\
\hline$\cdot, r$ & $1, r$ & $|\wedge|$. & הTr & T110r & rVq & 114.9 & $r_{\cdot}, \cdot$ & 07. & $r .17$ \\
\hline$\cdot, 7$ & 1,7 & 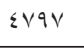 & $|r| 9 \mid$ & Vras & or & $1 . \Delta T 1$ & $r \cdot, \Lambda$ & ort & $r .1 V$ \\
\hline$\cdot, 7$ & 1,7 & $0 \leqslant 1 \leqslant$ & $1 \varepsilon \cdot r V$ & ATाז & $7 .$. & $1 . r V V$ & $r_{1},$. & $\sum q \pi$ & $r .1 \Lambda$ \\
\hline$\cdot, \Lambda$ & 1,9 & $r \wedge \leqslant \wedge$ & $701 \leqslant$ & rolo & TYA & TVYI & $r \cdot, \Lambda$ & TrI & المتوسط \\
\hline
\end{tabular}

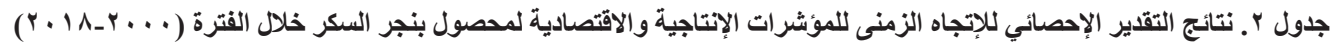

\begin{tabular}{|c|c|c|c|c|c|c|c|}
\hline معلد النمو & معنوية & معنوية & $\mathbf{F}$ & $\mathbf{R}^{r}$ & النموذج المقدر & المتغير & م \\
\hline$\% 1$ & $\left(I V, \cdot Y Y^{* * *}\right.$ & $(10,10)^{* * *}$ & $\curlyvee \wedge 9, \Upsilon^{* * *}$ & $\cdot, 90$ & $\ln \hat{Y}=105.3+0.100 X i$ & المساحة بالالف فدان & 1 \\
\hline - & $(1,1 \pi)^{-}$ & (\urcorner$), \vee \wedge) * *$ & $1, Y \Lambda_{-}$ & $\cdot, \cdot V$ & $\ln \hat{Y}=20.48+0.002 X i$ & الإنتاجية بالطن/ فدان & $r$ \\
\hline$\% 1 \cdot, r$ & $(1 \leq, Y T)^{* * *}$ & $(10,7 \pi)^{* *}$ & $r \leqslant \varepsilon, \varepsilon^{* *}$ & $\cdot, 9$ ? & $\ln \hat{Y}=2155.9+0.102 X i$ & الإنتاج بالالف طن & $r$ \\
\hline$\%$. & $(17,79)^{* *}$ & $(10, \leqslant 7)^{* *}$ & $r \vee \Lambda, Y^{* * *}$ & $\cdot, 9 \leqslant$ & $\ln \hat{Y}=88.21+0.100 \mathrm{Xi}$ & السعر المزرعي بالجنيه/طن & $\varepsilon$ \\
\hline$\% 9,9$ & $(Y \cdot, \cdot V)^{* * *}$ & $(11,79)^{* *}$ & $\varepsilon \cdot r, Y^{* *}$ & $\cdot, 97$ & $\ln \hat{Y}=1091.4+0.099 \mathrm{Xi}$ & التكاليف الكلية بالجنيه/ فدان & 。 \\
\hline$\% 1$ & $(I r, \wedge I)^{* * *}$ & $(11, r 1)^{* *}$ & $17 \varepsilon, r^{* * *}$ & $\cdot, 9$. & $\ln \hat{Y}=2053.6+0.100 X i$ & إجمالى العائد جنيه/فدان & 1 \\
\hline$\% r, r$ & $(7, \cdot 9)^{* *}$ & $(\varepsilon, 0 \vee)^{* *}$ & $r V, I^{* *}$ & $\cdot, \mathrm{v} \cdot$ & $\ln \hat{Y}=660.6+0.123 \mathrm{Xi}$ & صافي العائد بالجنيه/ فدان & $\mathrm{v}$ \\
\hline- & $(-\cdot, 19)^{-}$ & $(\wedge, 9 \cdot)^{* *}$ & - & $\cdot, \ldots r$ & $\ln \hat{Y}=1.91-0.002 \mathrm{Xi}$ & نسبة الإير اد إلى التكاليف جنية/فدان & $\wedge$ \\
\hline - & $(\cdot, \vee \vee)^{-}$ & $(\varepsilon, 19)^{* *}$ &., $079-$ & $\cdot, \cdot r$ & $\ln \hat{Y}=0.647-0.016 \mathrm{Xi}$ & العائد على الجنيه المستثر جنيد/فدان & 9 \\
\hline
\end{tabular}




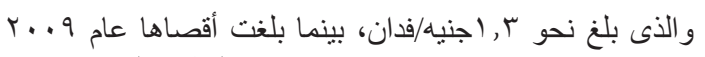

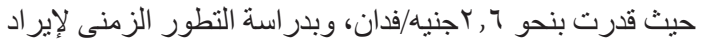

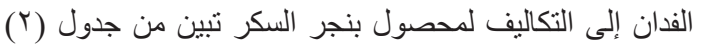

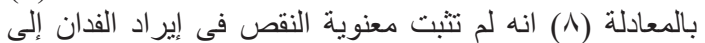

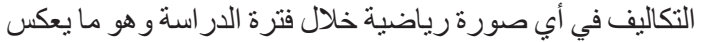
أن البيانات تدور حول متوسطها (ثبات نسبي).

9ـ تطور العائد على الجنيه المستثمر للفدان من بنجر السكر:

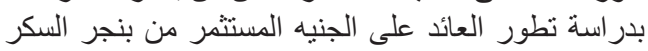

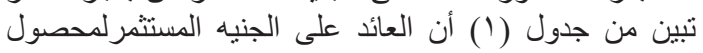

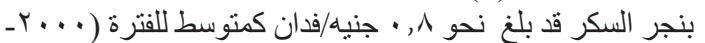

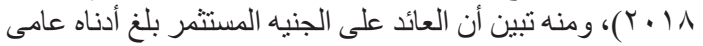

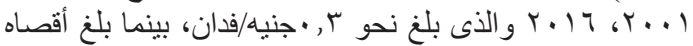

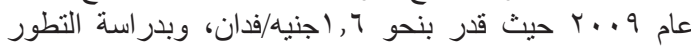

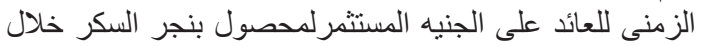

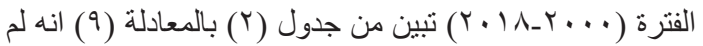

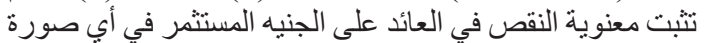

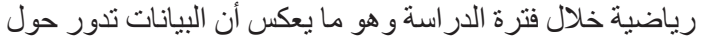
متوسطها (ثبات نسبي).

ثنانياً: التوزيع الجغرافي للطاقة الإنتاجية والمساحة لمحصول بنجر السكر على مستوى الجمهورية: يعتبر محصول بنجر السكر من محاصيل المناطق المهر البادرة

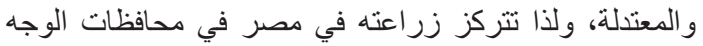

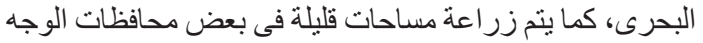

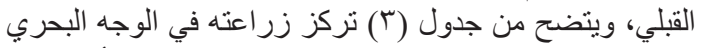

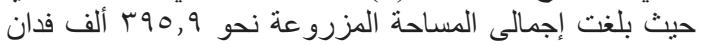

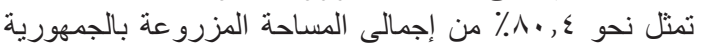

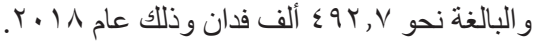

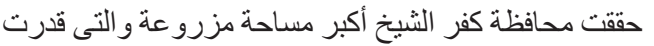

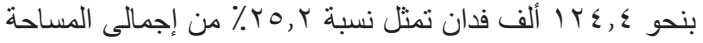

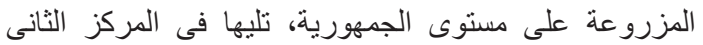

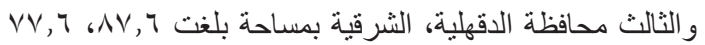

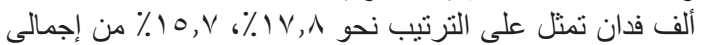

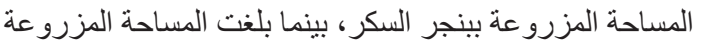

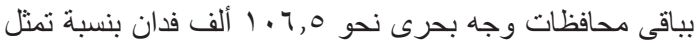

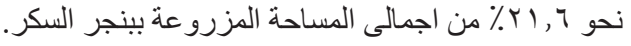

بلغت المساحة المزروعة ببنجر السكربمحافظات مصر

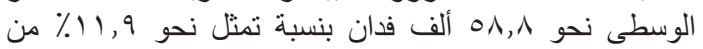

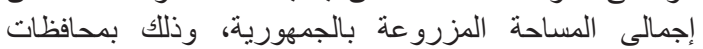

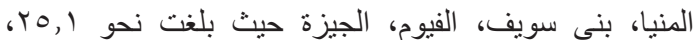

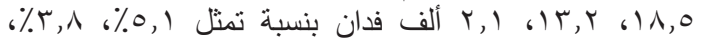

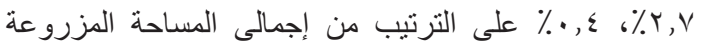

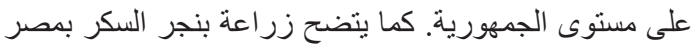

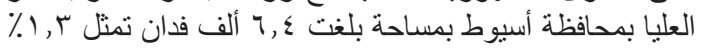

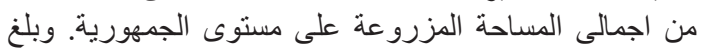

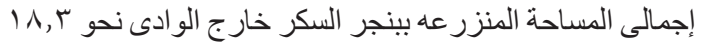

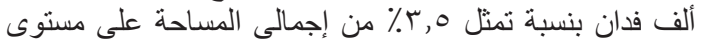

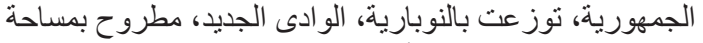

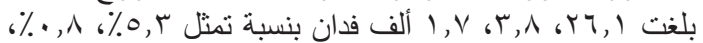
ب, ، ٪ من إجمالى المساحة على مستوى الجمهورية على الترتيب.

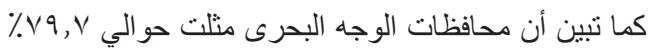

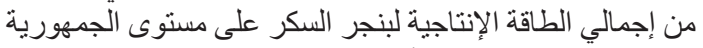

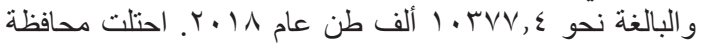

عـ تطور السعر المزرعي لبنجر السكر: بدر اسة تطور السعر المزر لبزعى لبنجر البكر السكر تبين من جدول

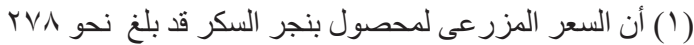

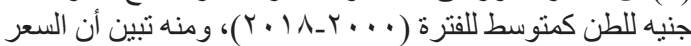

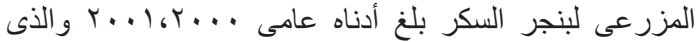

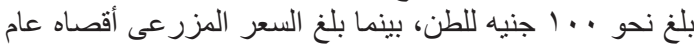

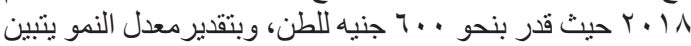

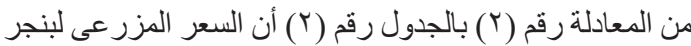

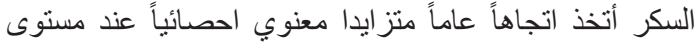

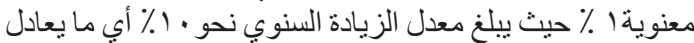
جن TV,N

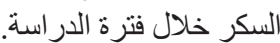

هـ تطور التكاليف الكلية لإنتاج بنجر السكر:

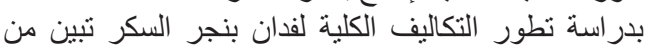

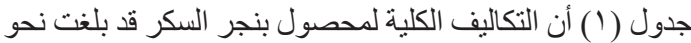

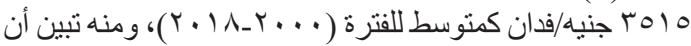

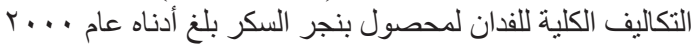

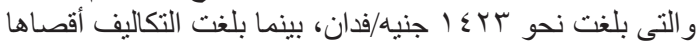

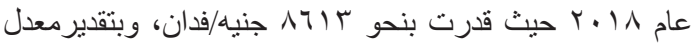

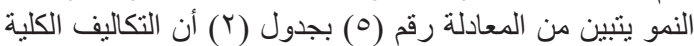

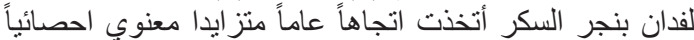

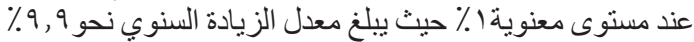

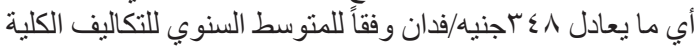
لإنتاج بنجر السكر خلال فترة الدراسة

بدر اسة تطور إجمالى العائد لفدان بنجر السكر ثبين من جدول

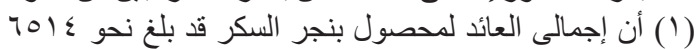

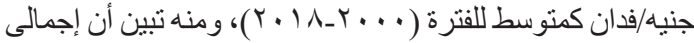

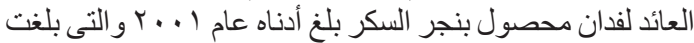

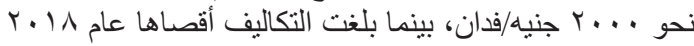

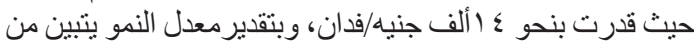

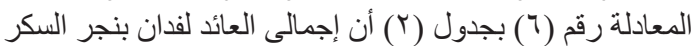

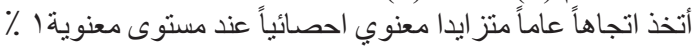

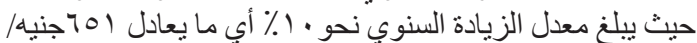

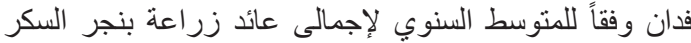

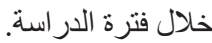

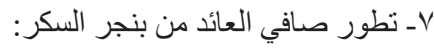

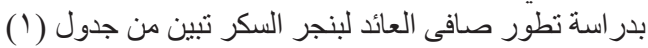

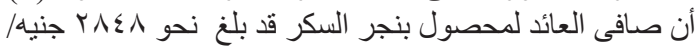

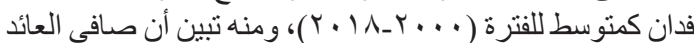

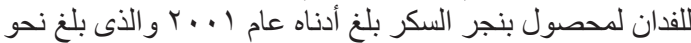

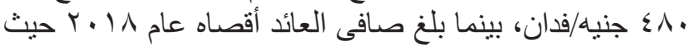

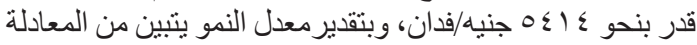

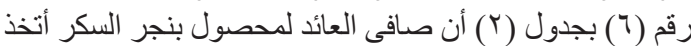

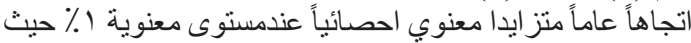

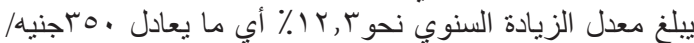

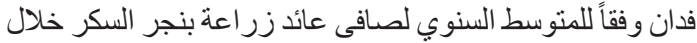

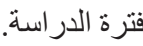

^-تطور إير اد الفدان إلى التكاليف من بنجر السكر:

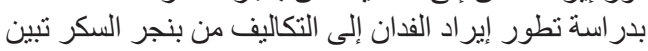

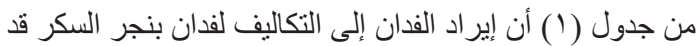

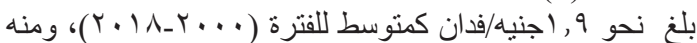

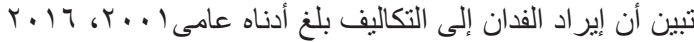

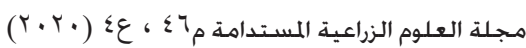


قطاع مصر العليا حو الى . , ب\% فقط من إجمالي الطاقة الإنتاجية لبنجر السكر على مستوى الجمهورية.

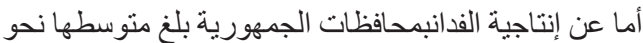

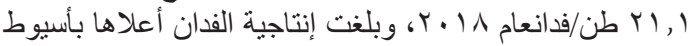

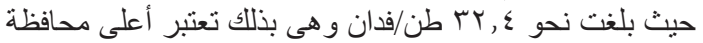

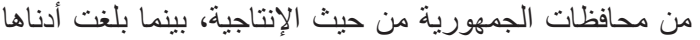

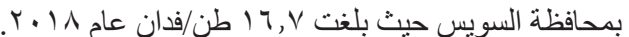

كفر الثيخ المرتبة الأولي تليها الدقطلية، الثرقية من حيث الطاقة

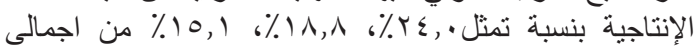

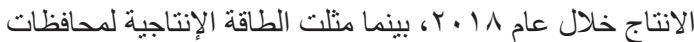

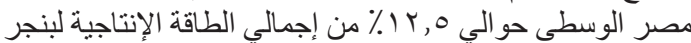

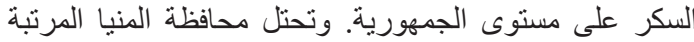
الأولى بين تلك الدحافظات من حيث الطاقة الإنتاجية بنسبية 1, \% ب، بينما بلغت الطاقة الإنتاجية لمحافظة أسيوط و التى مثلت

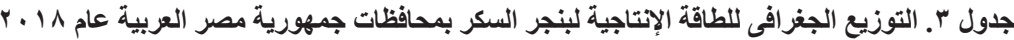

\begin{tabular}{|c|c|c|c|c|c|}
\hline الانتاجية طن/فدان & الأهمية النسبية & الف طن الإنتاج & $\begin{array}{c}\text { الأهمية النسبية } \\
\text { \% }\end{array}$ & الف فدانة & المحافظة \\
\hline$r \cdot, l$ & $r \varepsilon, \cdot$ & $r \leq 9 \leq, \varepsilon$ & $r_{0, r}$ & $\mid r \leq, r$ & كفر الثــــخ \\
\hline$r, r$ & $1 \wedge, \wedge$ & $19 \leqslant 9, \varepsilon$ & $\mid v, \Lambda$ & $\Lambda V, T$ & الدقهــــــــلية \\
\hline$r \cdot r$ & 10,1 & $10 \times 1, \varepsilon$ & $10, \mathrm{~V}$ & $V V, T$ & الثــــــــــرقية \\
\hline$r_{\cdot}, q$ & $\wedge$, & $\lambda r \varepsilon, v$ & $\wedge, 1$ & $r 9,9$ & البحي.ـــــــــة \\
\hline$r_{\cdot}, \tau$ & 0,7 & $0 \wedge \cdot, V$ & $0, V$ & $r \wedge, r$ & بور ســـــعيد \\
\hline$r \leqslant, r$ & $r, \varepsilon$ & rol,0 & $r, \cdot$ & $1 \leqslant, 0$ & الغربيــــــــــــة \\
\hline$r \cdot, \lambda$ & $1, v$ & $\mid \vee \wedge, V$ & $1, v$ & $\Lambda, 7$ & الاســــاعيلية \\
\hline 19,1 & 1,0 & 10r, & 1,7 & $v, v$ & الاســـكندرية \\
\hline$r_{\cdot}, \tau$ & 1,1 & 111,1 & 1,1 & $0, \varepsilon$ & دميـــــــــاط \\
\hline$r, r$ & $\cdot, \varepsilon$ & r & $\cdot, \varepsilon$ & $1, v$ & المنوفيــــــــــة \\
\hline $19,$. & $\cdot, 1$ & 0,7 & $\cdot, 1$ & $\cdot, r$ & القليوبيـــــــــــة \\
\hline $17, V$ & $\cdot, \cdot$ & $1, v$ & $\cdot, \cdot$ & $\cdot, 1$ & الســــــويـــس \\
\hline$r \cdot, q$ & $v q, v$ & Аrтq, & $\Lambda \cdot, \varepsilon$ & $r 90,9$ & جملة الوجه البحري \\
\hline$r T, r$ & 0,7 & $0 \wedge 1, \varepsilon$ & 0,1 & $r_{0,1}$ & 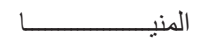 \\
\hline $19, \varepsilon$ & $r, 0$ & ron,, $\mathrm{V}$ & $r, \wedge$ & 11,0 & الفيــــــــــوم \\
\hline$r r, q$ & $r$, & $r \mid \leq, r$ & $r, r$ & $1 r, r$ & بني سويف \\
\hline$r, T$ & $\cdot, \varepsilon$ & $\leq 7,0$ & $\cdot, \varepsilon$ & $r, 1$ & 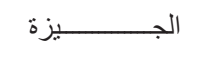 \\
\hline$r, 1$ & Ir,o & $1 r \ldots, q$ & 11,9 & $0 \wedge, \wedge$ & جملة مصر الوسطي \\
\hline$r, \varepsilon$ & $r_{,} \cdot$ & $r \cdot 0, \lambda$ & $1, r$ & $7, \varepsilon$ & أســـــــــيوط \\
\hline rr, & $r, \cdot$ & $r \cdot 0, \lambda$ & $1, r$ & $7, \varepsilon$ & جملة مصر العليا \\
\hline$r, r$ & $9 \varepsilon, r$ & q४vo, V & 94,7 & $\leq 71,1$ & إجمالى داخل الو ادى \\
\hline 19, & $\varepsilon, \lambda$ & $\leq 97,9$ & $0, r$ & $r 7,1$ & النوباريـــــــــــة \\
\hline $1 \wedge, V$ & $\cdot, V$ & $v, r$ & $\cdot, \wedge$ & $r, \Lambda$ & الو ادي الجديد \\
\hline$r_{\cdot}, \cdot$ & $\cdot, r$ & ع ع & $\cdot, r$ & $1, v$ & مطـــــــــروح \\
\hline $19,$. & 0,1 & $7 \cdot 1,7$ & $7, \varepsilon$ & $r, T$ & جملة خارج الو ادي \\
\hline$r 1,1$ & $1 \cdots$, & $1 \cdot r V V, \varepsilon$ & $1 \cdots$, & $\sum q r, V$ & إجمالي الجمهورية \\
\hline
\end{tabular}

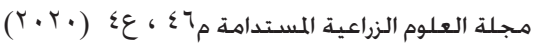


من السكر، وتطور نصيب الفرد فى مصر من السكر، وقد تم

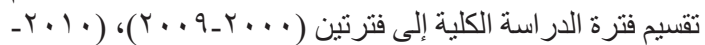

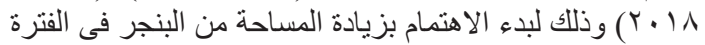

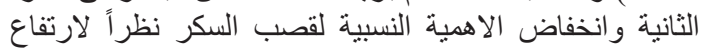
الاحتباجات المائية له.

ا ـ تطور إجمالى إنتاج السكر فى مصر :

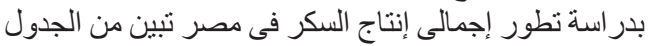

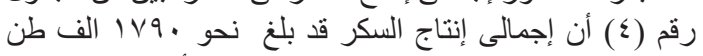

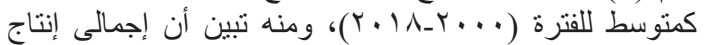

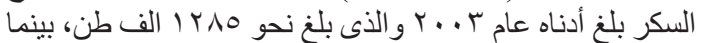

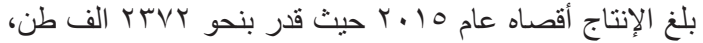

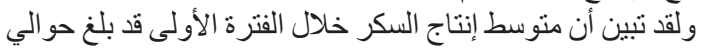

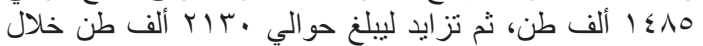

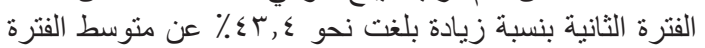

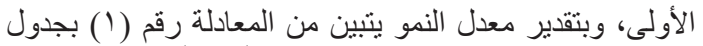

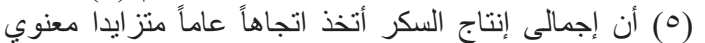

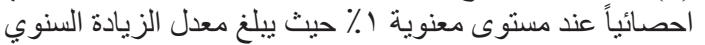

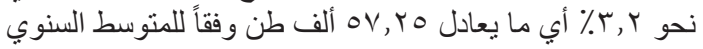

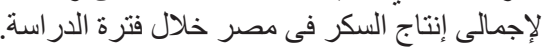

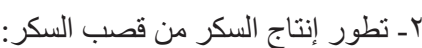
بدر اسة تطور إنتاج السكر من قصب النباج السكرتبين من جدول

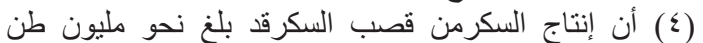

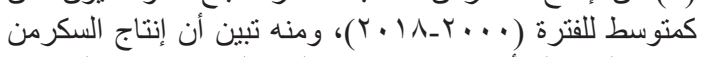

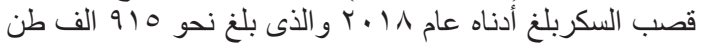

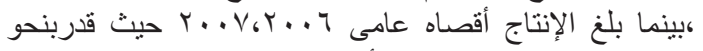

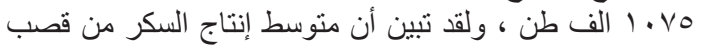

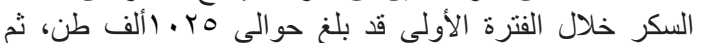

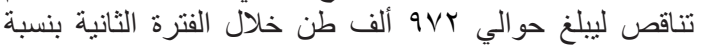

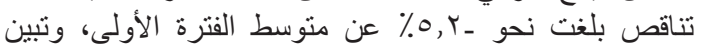

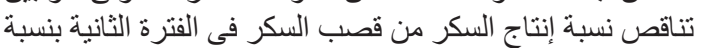

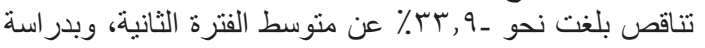

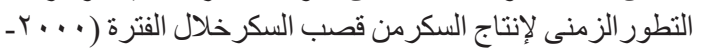

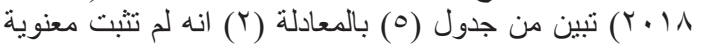

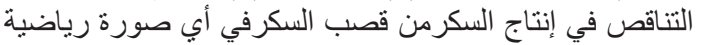

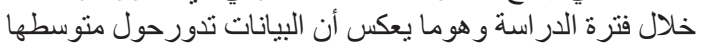

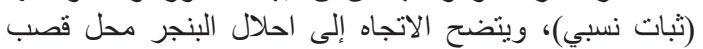

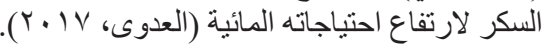

$$
\text { rـ تطور إنتاج السكر من بنجر السكر: }
$$

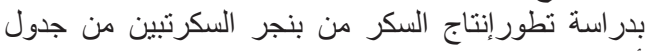

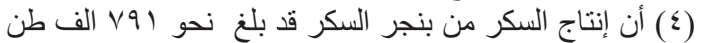

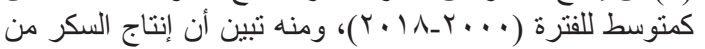

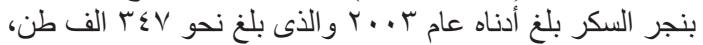

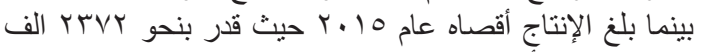

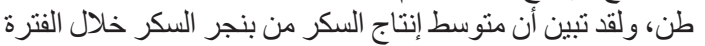

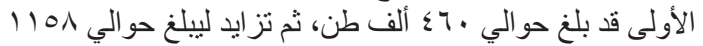

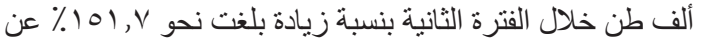

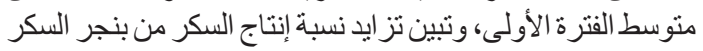

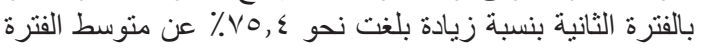

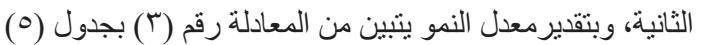

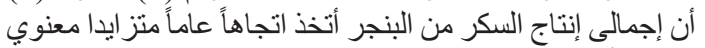

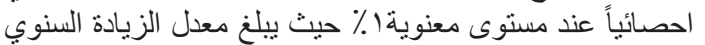

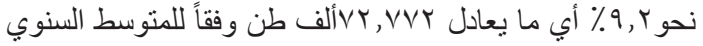
لإنتاج السكر من بنجر السكر فى مصر خلال فترة الدر اسة.
ثالثاً: تأثير المناطق الجغر افية وظروف المناخ بها على إنتاجية

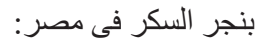

وبدراسة تأثير المنطفة الجغر افية وظروف الفيكرة المناخ بها على

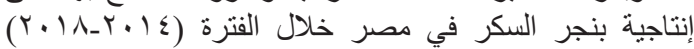

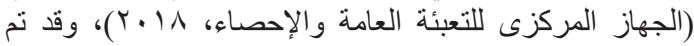

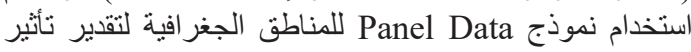

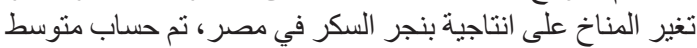

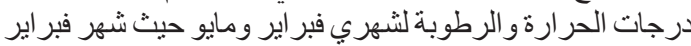

هو شهر تكوين خضري وشهر مايو الرطوبة هو شهر نضرج المحصول.

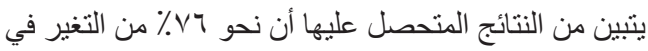

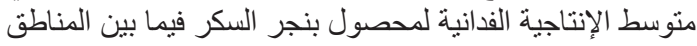

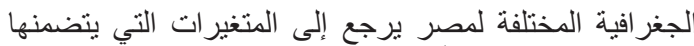

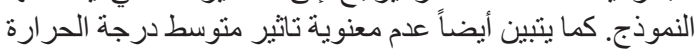

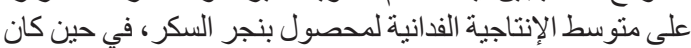

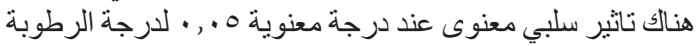

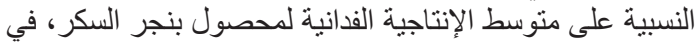

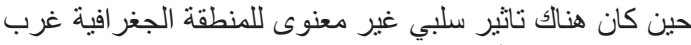

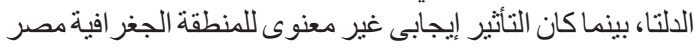

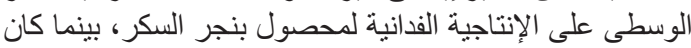

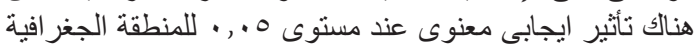

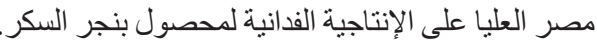

$\mathrm{Y}=6.91+0.93 \mathrm{X}_{1}-0.163 \mathrm{X}_{2}-0.38 \mathrm{D}_{1}+2.53$ $\mathrm{D}_{2}+3.71 \mathrm{D}_{4}$

(0.38) $(1.67)(-2.19)^{*}(-0.13)^{-}(0.96)^{-}(2.01)^{*}$

$$
\mathrm{R}^{-2}=0.76 \quad \mathrm{~F}=13.92 * *
$$

حيث:- تشير إلى إنتاجية الفدان من محصول بنجر السكر (طن/ فـان).

$$
\text { تشير إلى متوسط درجة الحر ارة العظمى X X }
$$$$
\text { تشير إلى متوسط الرطوبة النسبية X }
$$

= D 1 القيمة (1) في مشاهدات غرب الدلنا، ويأخذا القيمة ( • ) في مشاهدات باقي المناطق.

= D 2

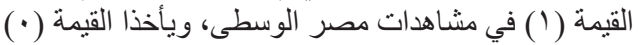
في مشاهدات باقي المناطق.

= D D $_{4}$ القيمة (1) في مشاهدات مصر العليا، ويأخذا القيمة ( • ( ) في مشاهدات باقي المناطق. * تنشير إلى معنوية المعالم عند مستوى معنوية 0.05.

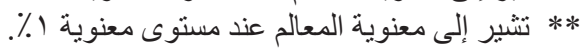

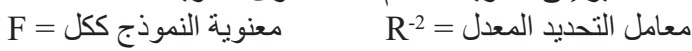
رابعاً: الوضع الر اهن لإنتاج السكر والاستهلاك الكلي و الاكتفاء الذاتي في جمهورية مصر العربية الإنية:

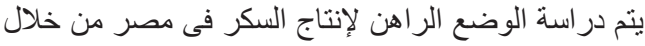

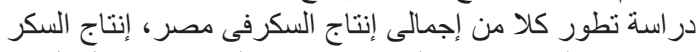

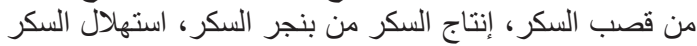

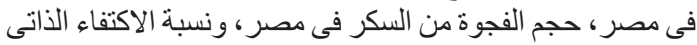

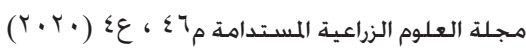




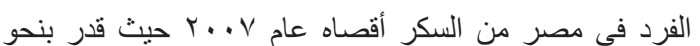
خلو 9

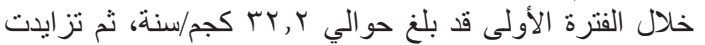

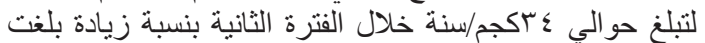

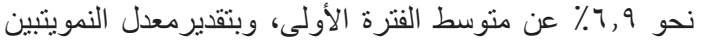

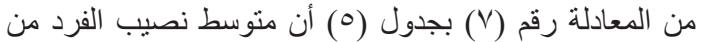

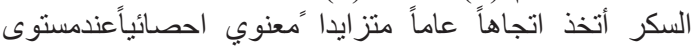

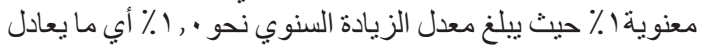

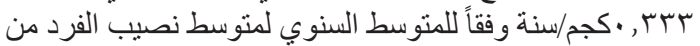
السكر فى مصر خلال فترة الدر اسة. لمنة

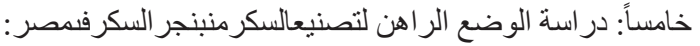

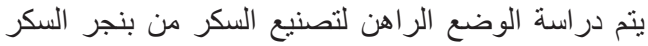

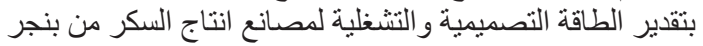

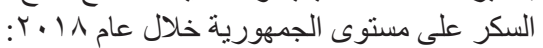

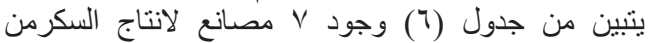

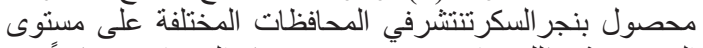

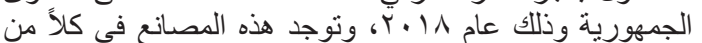

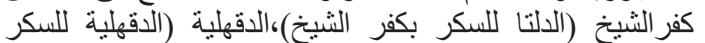

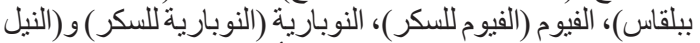

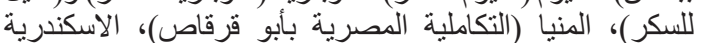

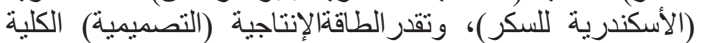

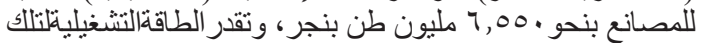

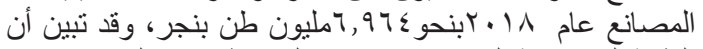

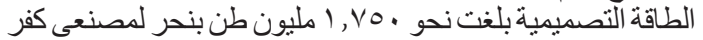

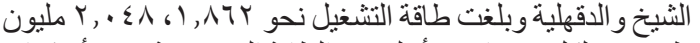

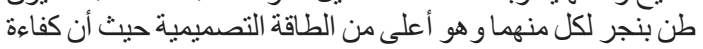

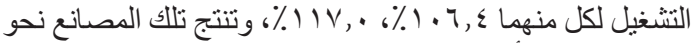

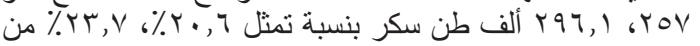

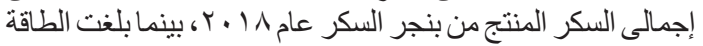

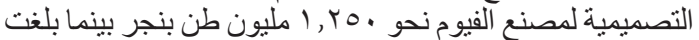

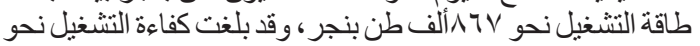

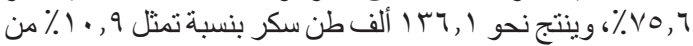
إجمالى السكر المنتج من بنجر السكر ، بينما بلغت الطاقة التصديمية

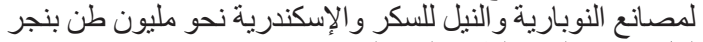

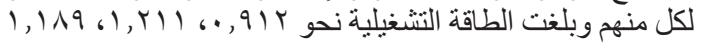

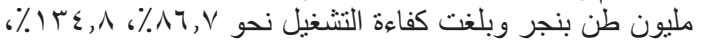

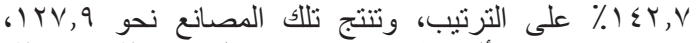

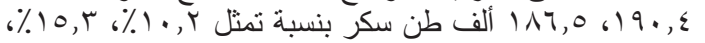

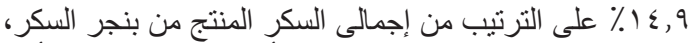

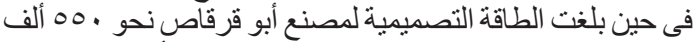

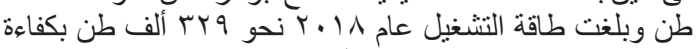

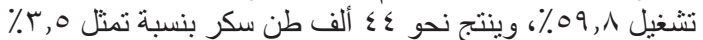
من إجمالى السكر المنتج من بنجر السكر.

سادساً: التقييم المالى و الاقتصادى لشركة الدلتا للسكر

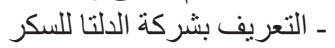
تقع شركة الدلتا للسكر بمحافظة كفر الثيخ، تأسست الثركة الثركة

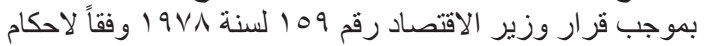

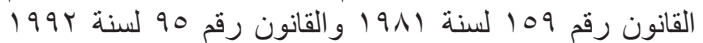

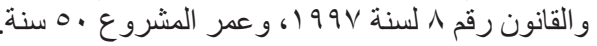

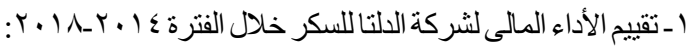

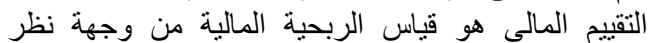

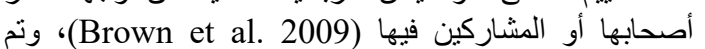

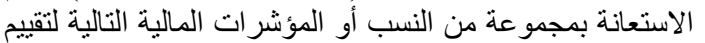

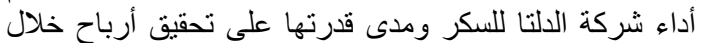

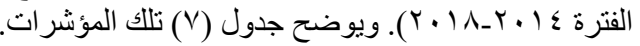

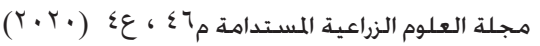

ع ـ تطور إجمالى استهلاك السكر فى مصر:

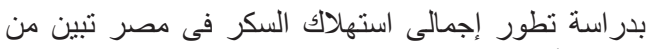

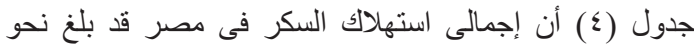

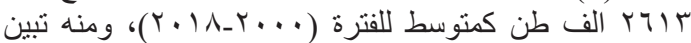

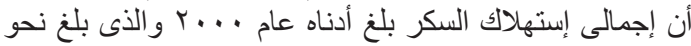

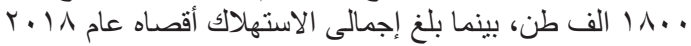

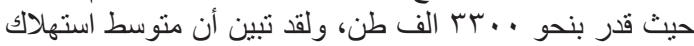

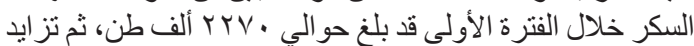

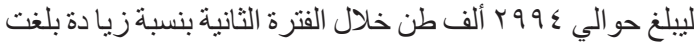

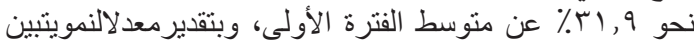

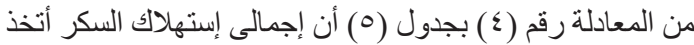

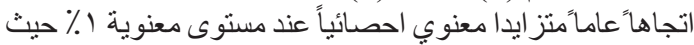

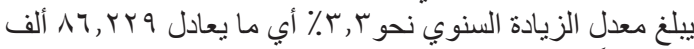

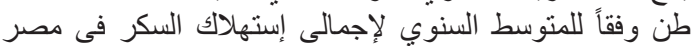
خلال فترة الدر اسة.

هـ تطور الفجوة السكرية فى مصر : بدراسة تطور الفجوة السكرية فى مصر الفر تبين من جدول

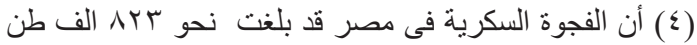

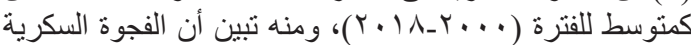

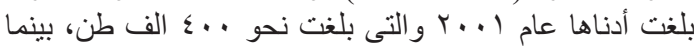

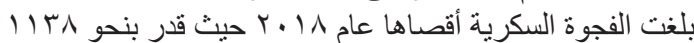
الف طن، ولقد تبين أن متوسط الفجوة السكرية خلال الفال الفترة

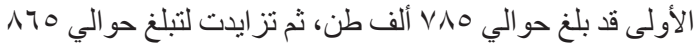

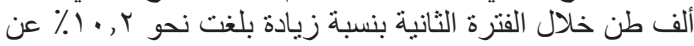

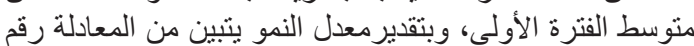

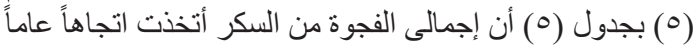

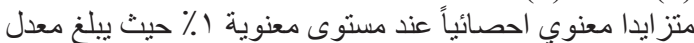

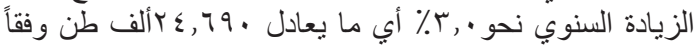

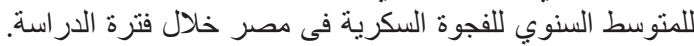

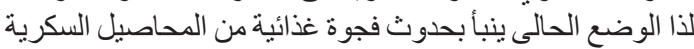

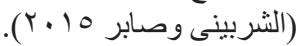

7ـ تطور نسبة الاكتفاء الذاتى من السكر:

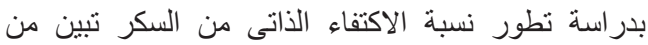

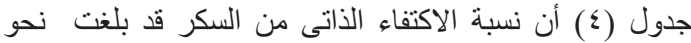

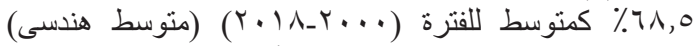

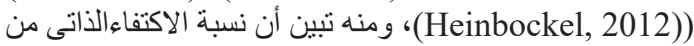

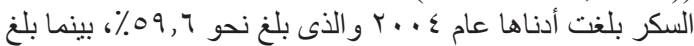

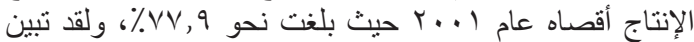

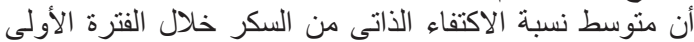

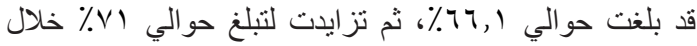

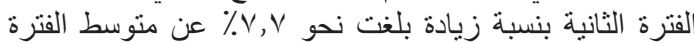

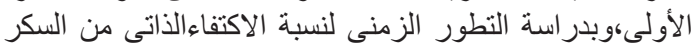

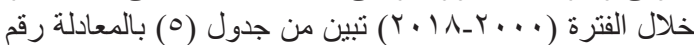

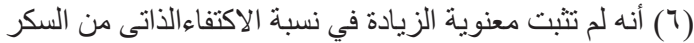

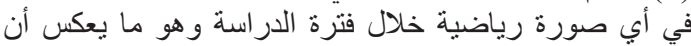
البيانات تدور حول متوسطها (ثبات نسبي).

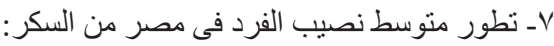

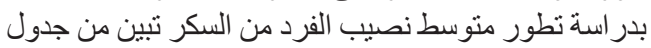

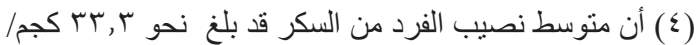

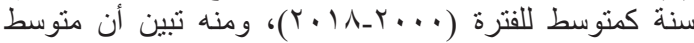

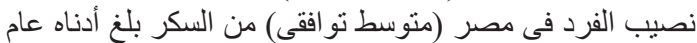

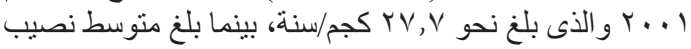


جدول ع. إنتاج السكر من قصب السكر وينجر السكر وإجمالى الإستهلاك ونسبة الاكتفاء الذاتى ومتوسط نصيب الفرد من السكر فى مصر خلال الفترة (r.lA_r...)

\begin{tabular}{|c|c|c|c|c|c|c|c|c|c|}
\hline متوسط & نسبة الاكتفاء & (الف طنوية & (الاستهلاكث & 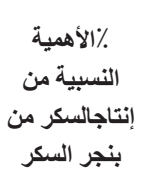 & 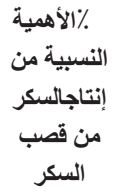 & إنتاج السكر & من قصب إنتاج السكر & (الفمالى إنتاج & السنة \\
\hline$r \wedge, l$ & $V V, \varepsilon$ & $\varepsilon .7$ & $1 \wedge \ldots$ & $r_{0,0}$ & $V \leqslant, 0$ & rot & $1 . r \Lambda$ & $1 \pi 95$ & $r \ldots$ \\
\hline$r v, v$ & $V V, q$ & $\varepsilon \cdots$ & 11.7 & $r \wedge, r$ & $v 1, \wedge$ & rqV & $1 \ldots 9$ & $1 \varepsilon .7$ & $r \ldots l$ \\
\hline$r$. & $7 \wedge, 7$ & 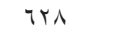 & $r \ldots$ & $r \wedge, q$ & $(1,1$ & एव५ & $9 \vee 7$ & ITVY & $r \ldots r$ \\
\hline$r \cdot, q$ & $71, r$ & 110 & r).. & $r v,$. & $V T,$. & $r \leqslant v$ & $9 \pi \wedge$ & IrNo & $r . . r$ \\
\hline rr, & 09,7 & 94. & r... & $r 7,9$ & $V T, l$ & rus & $1 \cdots r$ & ITV. & $r \ldots \varepsilon$ \\
\hline Tr, & $7 r, 0$ & ᄉT) & rron & $r \cdot, \cdot$ & $v_{\cdot}, \cdot$ & $\leq \leqslant 9$ & $1 . \leqslant 1$ & $1 \leq 9 V$ & $r \ldots o$ \\
\hline$r \varepsilon, \varepsilon$ & דיד & $9 \ldots$ & $r \leqslant V_{0}$ & $r 1,9$ & 71,1 & $0 . r$ & $1 . V r$ & $10 \% 0$ & $r \ldots r$ \\
\hline$r \varepsilon, q$ & $T \wedge, 0$ & ᄉ). & roTh & rی, 9 & 71,1 & Th & 1.10 & IVON & $r \ldots r$ \\
\hline$r_{0,1}$ & 09,9 & 1.01 & YTE. & Tr,. & 71, & $0 . v$ & 1.10 & $101 \mathrm{r}$ & $r \ldots \Lambda$ \\
\hline$r \varepsilon, \varepsilon$ & $7 \cdot, 1$ & $1 \cdot \varepsilon$. & ryo. & $r v, 1$ & $7 r, 9$ & $09 V$ & 1.15 & 171. & $r \ldots q$ \\
\hline$* r r, r$ & $* * 47,1$ & $\vee \wedge 0$ & rrv. & r., & $79,$. & $\leq 4$. & 1.10 & $1 \leqslant 10$ & متوسط الفترة \\
\hline$r \varepsilon, 1$ & $V \varepsilon, r$ & 719 & YTA. & $\leq 9, \vee$ & $0 ., r$ & 99. & $1 \ldots 1$ & 1991 & $r .1$. \\
\hline$r \varepsilon, 1$ & $79,$. & Nor & rVo. & $\leq \wedge, 1$ & 01,9 & 915 & 910 & 1191 & $r .11$ \\
\hline$r \varepsilon, Y$ & $v_{\cdot}, 1$ & 100 & rА૫. & $0 ., 1$ & $\leq 9,9$ & $1 \cdots \varepsilon$ & $1 \ldots 1$ & $r \ldots o$ & $r . M r$ \\
\hline$r \varepsilon, r$ & 71,9 & $9 . r$ & rq.. & or, 1 & $\leq 7,9$ & 1.7 & $94 \wedge$ & 1991 & $r .1 r$ \\
\hline$r \leq, 7$ & $V Y, 7$ & $V \cdot r$ & $r .$. & $00, \varepsilon$ & $\varepsilon \varepsilon, 7$ & ITVE & $1 \cdot r \varepsilon$ & rrqA & $r .1 \leq$ \\
\hline$r \varepsilon, \wedge$ & $V T, 0$ & VYA & r... & or,$\wedge$ & $\varepsilon r, r$ & $1 \pi \leqslant V$ & $1 . r o$ & TMVT & $r .10$ \\
\hline$r \varepsilon, v$ & 79,0 & $97 \leq$ & MIT. & ov, 7 & $\varepsilon r, \varepsilon$ & 1 1ro & 941 & T197 & $r .17$ \\
\hline$\Gamma \varepsilon$, & $v \cdot r$ & 901 & r... & 01,9 & $\{1,1$ & ITRO & $9 r \leq$ & $r r \leqslant 9$ & r.IV \\
\hline$r \varepsilon, \wedge$ & 70,0 & גז & ז... & $O V, V$ & $\varepsilon r, r$ & $1 T \leq A$ & 910 & rir. & $r .11$ \\
\hline *rs, & $* * \vee 1$, & 170 & Y११ะ & $0 \leqslant, \varepsilon$ & $\leq 0,7$ & 1101 & QVT & TाT. & متوسط الفترة \\
\hline שT,r & $* * \uparrow \wedge, 0$ & ArT & rוד & $\varepsilon \varepsilon, r$ & 00,9 & 199 & $1 \ldots$ & 189. & المتوسط العام \\
\hline
\end{tabular}

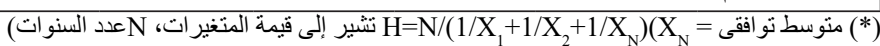

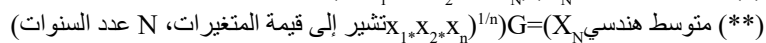
المصدر : وزارة الزر اعة واستصلاح الأر اضیى، مجلس المحاصسل السكرية، المحاصيل السكرية و إنتاج السكر فى مصر واعلى والعالم، التقرير السنوى، أعداد مختلفة.

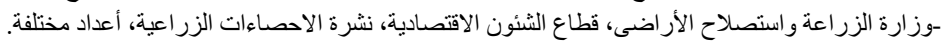

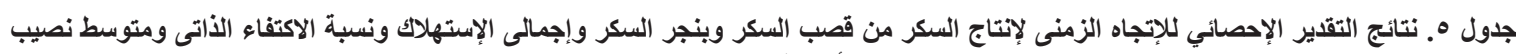

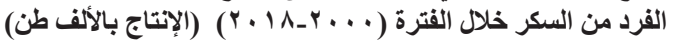

\begin{tabular}{|c|c|c|c|c|c|c|c|}
\hline معدل النمو & معنوية & معنوية & $\mathbf{F}$ & $\mathbf{R}^{2}$ & النموذج المقر & المتغير & م \\
\hline$\% \Gamma, \varepsilon$ & $(11, \times 1)^{* * *}$ & $($ (१, १^)** & $I r V, Y^{* * *}$ & $\cdot, \wedge \wedge$ & $\ln \hat{Y}=1246.3+0.034 \mathrm{Xi}$ & إجمالى الإنتاج & 1 \\
\hline- & $(1,00)^{-}$ & $(\varepsilon r, r \Gamma)^{* * *}$ & $r, \varepsilon-$ & $\cdot, 1 T$ & $\ln \hat{Y}=1034.1-0.003 \mathrm{Xi}$ & إنتاج السكر من قصب السكر & 2 \\
\hline$\%, r$ & $(\mid r, \wedge r)^{* * *}$ & $(1 \Gamma, \wedge 0)^{* * *}$ & $191, r^{* * *}$ & $\cdot, 94$ & $\ln \hat{Y}=282.4+0.092 \mathrm{Xi}$ & إنتاج السكر من بنجر السكر & 3 \\
\hline$\% r, r$ & $(\mid V, 1 \cdot)^{* * *}$ & $(\Sigma 7,79)^{* * *}$ & rar, O** & $\cdot, 9 \leq$ & $\ln \hat{Y}=1865.4+0.032 \mathrm{Xi}$ & إجمالى الاستهلاك (الف طن) & 4 \\
\hline$\%$ \% & $(\curlyvee, १ \wedge)^{* * *}$ & $(\wedge, \wedge 0)^{* *}$ & $\wedge, 9^{* * *}$ & $\cdot, r \cdot$ & $\ln \hat{Y}=587.4+0.030 \mathrm{Xi}$ & الفجوة السكرية (الف طن) & 5 \\
\hline- & $(\cdot, 07)^{-}$ & $(Y r, 1 T)^{* * *}$ & $\cdot, \Gamma$ & $\cdot, \cdot \leq$ & $\ln \hat{Y}=66.8+0.002 \mathrm{Xi}$ & نسبة الاكتفاء الذاتى ٪ & 6 \\
\hline$\% 1$, & $(\varepsilon, T Y)^{* * *}$ & $(\varepsilon 1,7 \leqslant)^{* * *}$ & $r_{1}, \varepsilon^{* * *}$ & $\cdot$, or & $\ln \hat{Y}=30.1+0.010 \mathrm{Xi}$ & متوسط نصيب الفرد من السكر (كجم/ & 7 \\
\hline
\end{tabular}




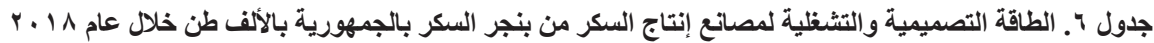

\begin{tabular}{|c|c|c|c|c|c|c|c|c|}
\hline الإنتاجية & $\begin{array}{c}\text { الفكران من طن اتنج } \\
\text { الدان }\end{array}$ & الإنتاج السبية الاهمية & كمية السكر & التشغيل٪\% & 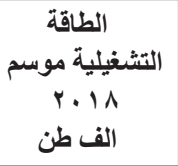 & التصميمية الفة الفة & تاريخ الإنشاء & المصنع \\
\hline$\cdot, 1$ r & $r, r$ & $r_{\bullet}, T$ & rov,. & $1 \cdot 7, \varepsilon$ & INTY & ivo. & 1914 & الدلتا للسكر بكفر الثيخ \\
\hline$\cdot, 1 \leq 0$ & $r, r$ & $r T, V$ & r97, 1 & $11 \mathrm{~V},$. & $r \cdot \leqslant \Lambda$ & IVo. & 1997 & الدقهلية للسكر ببلقاس \\
\hline$\cdot, 1 \leq \leq$ & $r, r$ & $1 \cdot, 9$ & $\mid r 4,1$ & $v_{0, T}$ & $9 \leqslant 0$ & ro. & r... & الفيوم للسكر ببلقاس \\
\hline$\cdot, 1 \leq \Lambda$ & $r, \varepsilon$ & $1 \cdot, r$ & $1 r v, q$ & $\wedge 7, \mathrm{~V}$ & NTV & $1 \ldots$ & r... & النوبارية للسكر \\
\hline$\cdot, 1 \leq 1$ & $r, q$ & $10, r$ & $19 \cdot, \varepsilon$ & $1 \Psi \varepsilon, \lambda$ & 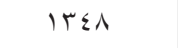 & $1 \ldots$ & $r \cdot 1$. & النيل للسكر بالنوبارية \\
\hline$\cdot, 1 T \leq$ & $r, \Lambda$ & $r, 0$ & $\varepsilon \varepsilon,$. & 09,1 & rrq & 00 . & 1991 & التكاملية المصرية \\
\hline$\cdot,|M|$ & $r, r$ & $1 \leqslant, 9$ & 1117,0 & $1 \leq r, V$ & $1 \leqslant r V$ & $1 \ldots$ & r. & الأسكندرية للسكر \\
\hline$\cdot, 1 \vee 9$ & $r, r$ & $1 \ldots$ & $\mid r \leqslant \wedge,$. & $1.7, r$ & 797 & 700. & & الاجمالى \\
\hline
\end{tabular}

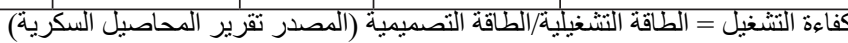

الكفاءة الإنتاجية = كمية السكر المنتج/كمية بنجر السكر الموردة (المصدر تقرير المحاصيل المبليل السكرية)

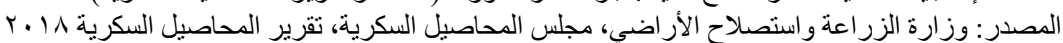

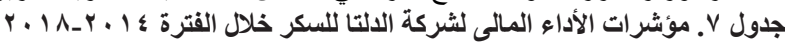

نسبة الفوائد إلى المبيعات: وتثشير إلى ما تستنزفة الفوائد

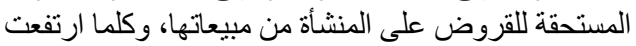

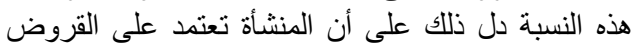

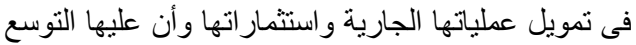

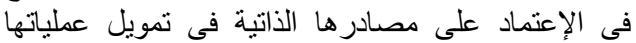

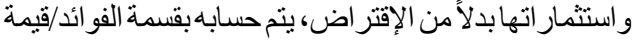

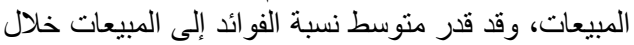

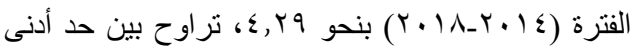

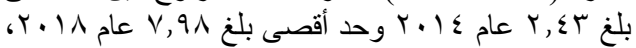
ويرجع هذا الارتفاع النسبى إلى إرتفاع قيمة المبيع التيعات بالنسبة

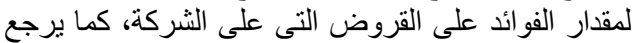
تذبذب هذه النسبة إلى إختلاف النسبة بينهما.

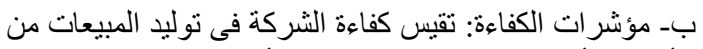
الأصول، أى كفاءة الإدارة فى تشغيل الفيل الأصول.

معدل دوران إجمالى الأصول: يتم حسابه بقسمة قيمة المبيعات/

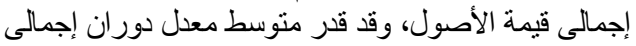

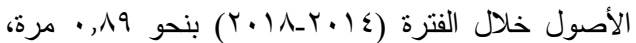

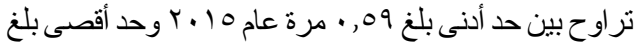

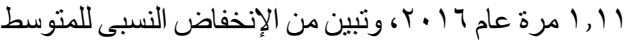

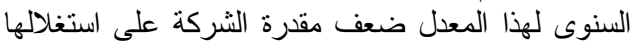
أصولها استغلالاً اقتصادياً فى توليد مبيعات منها.

معدل دوران الأصول الثابتة: يتم حسابه بقسمة قيمة

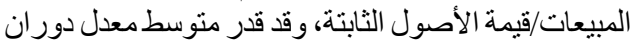

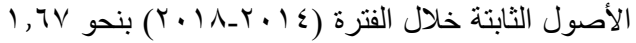

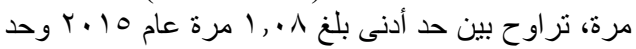

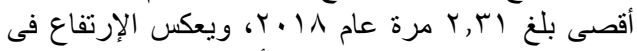

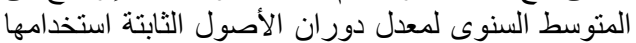
فى العملية الإنتاجية وتوليد المبيعات منها.

معدل دوران الأصول المتداولة: ينم حسابه بقسمة قيمة

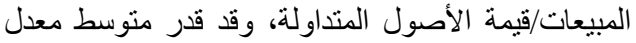

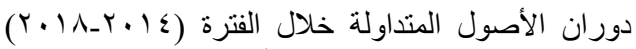

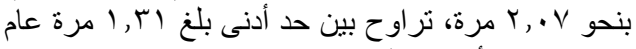

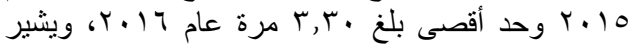

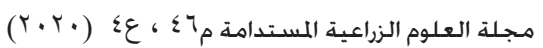

أـ مؤشر ات السيولة و الجدارة الإئتمانية: توضح قدرة الثركة في مقابلة إلتزاماتها الجارية بمالتها لديها

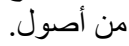

نسبة التداول: تستخدم لمعرفة قدرة المنشأة على مقابلة

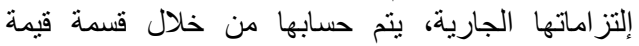

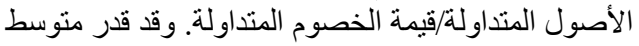

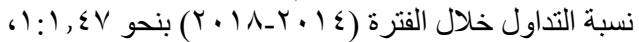

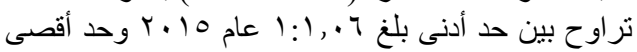

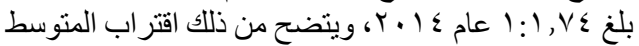

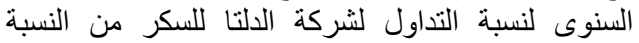

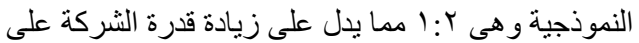

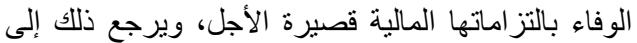
الارتفاع النسبى فى بنود الأصول المتداولة للثشركة.

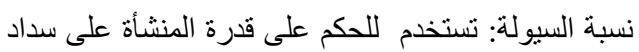

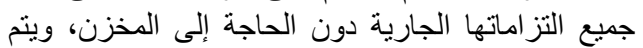

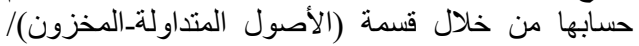

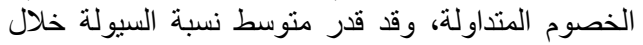

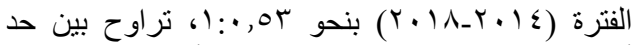

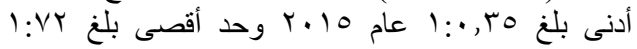

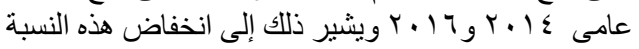

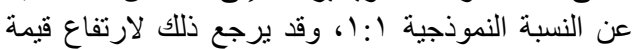

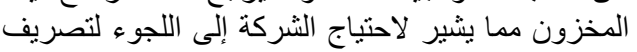
المخزون لزيادة السيولة لسداد التز اماتها الجارية.

معدل تغطية الأصول الثابتة: يقيس عدد المر ات التى يمكن

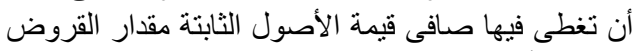

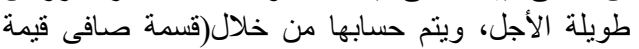

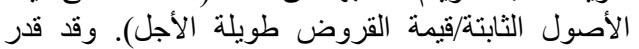

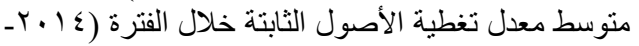

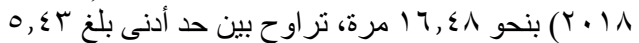

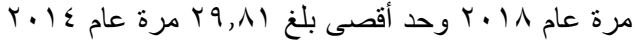

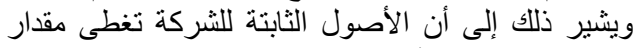

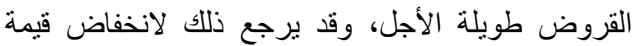
القروض طويلة الأجل وارتفاع قيمة الأصول الثابتة لأنفاض. 
إنخفضت هذه النسبة زالت قدرة المنشأة على مواجهة

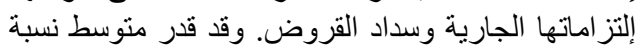

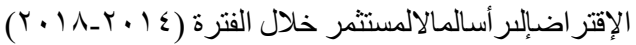

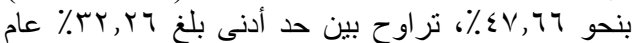

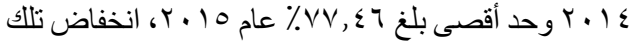

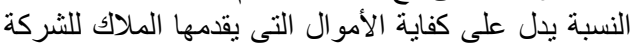
حتى تقابل التز اماتها الجارية.

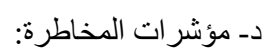

تقبس المخاطرة المالية ومقدرة المنشأة على استخدام القروض لفائدة المساهمين.

- - نسبة الإقتراض إلى إجمالى الأصول: يتم حسابه بقسمة

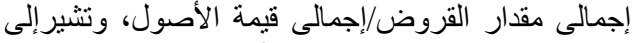

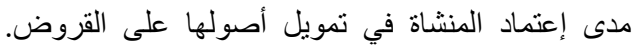

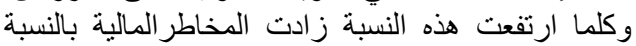

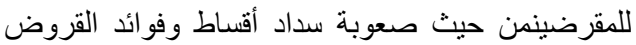

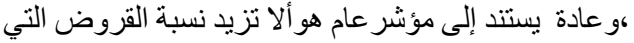

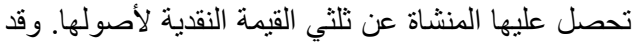

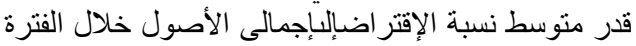

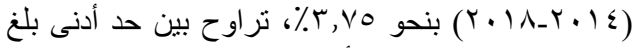

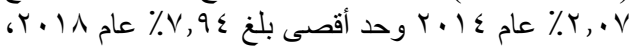
ويشير ذلك إلى تمتع الثركة بنسبة معتدلة بين الإقتر اضل عاض

$$
\text { و إجمالى أصولى لهاب. }
$$

نسبة الإقتر اض إلى حقو قا لملكية: يتم حسابه بقسمة إجمالى

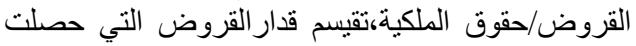

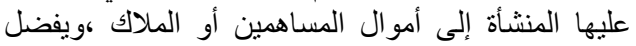

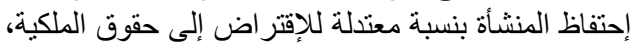

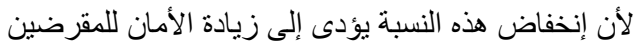

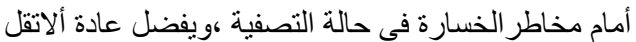

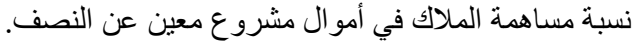

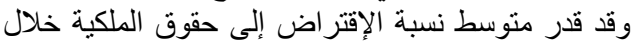

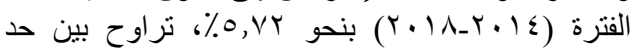

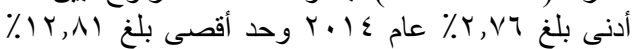

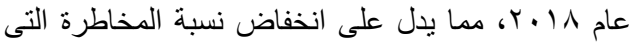
يو اجهها الدائنون.

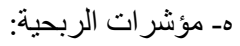
تقيس مؤشرات الربحية قدرة المنشأة على تحقيق الأرباح لتعكس بذلك دورمختلف السياسات التي تتخذها المنشأة.

العائد على المبيعات: يتم حسابه بقسمة صافى الربح قبل العبل

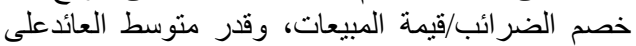

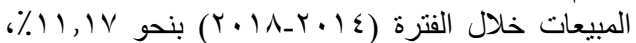

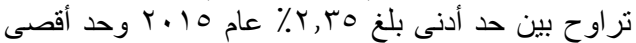

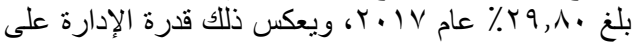

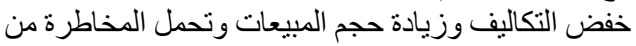

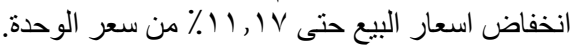

العائد على حقوق الملكية: يتم حسابه بقسمة الأرباح بعد

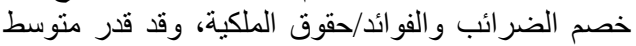

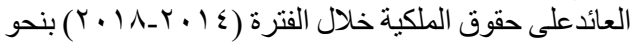

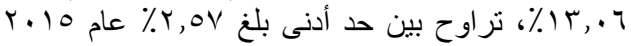

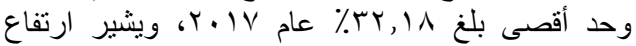

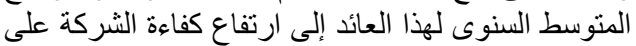
تحقيق ارباح مرتفعة على حقوق ملكيتها.
ارتفاع المتوسط السنوى لهذا المعدل إلى كفاءة الثركة

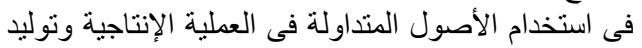

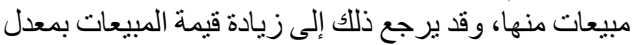
أكبر من الزيادة فى قيمة الأصول المتداولة لئه

معدل دور ان حقوق الملكية: يتم حسابه بقسمة قيمة المبيعات/

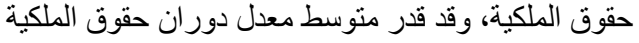

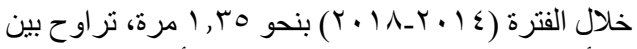

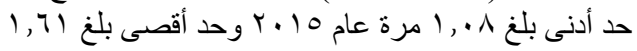

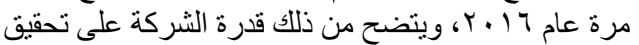
عائد مرتفع على حقوق ملكيتها.

معدل دوران رأس المال المستثمر: يتم حسابه بقسمة قيمة المانة

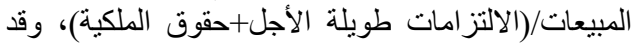

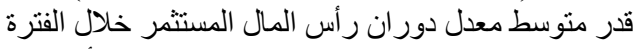

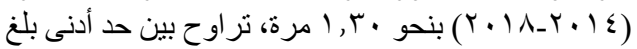

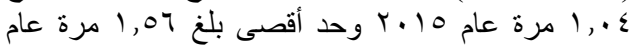

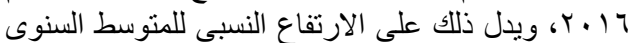

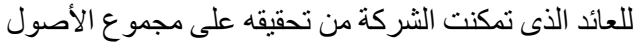

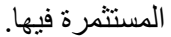

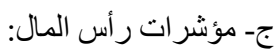
تقيس مدى إسهام كل من الملاك ول الدائنين فى تمويل المنشأة. نسبة الأصول الثابتة إلى حقوق الملكية: يتم حسابه بقسمة

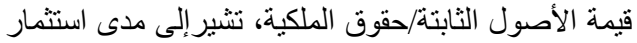

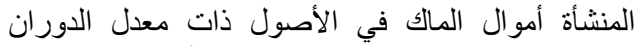

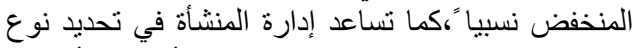

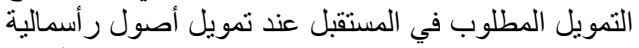

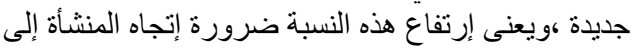

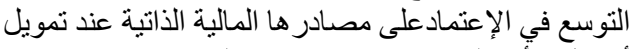

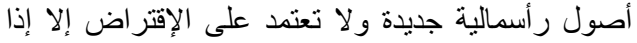

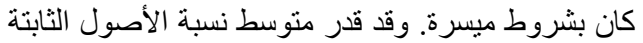

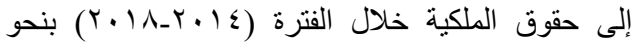

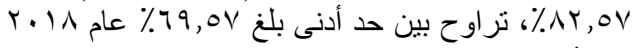

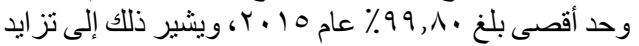

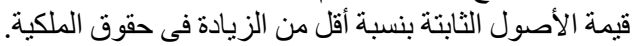

نسبة الخصوم المتداولة إلى حقوق الملكية: يتم حسابه بقسمة

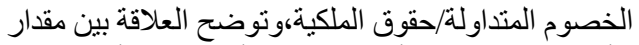

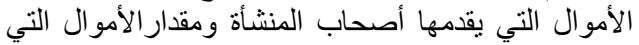

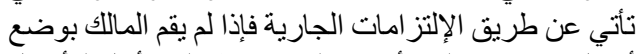

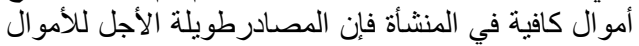

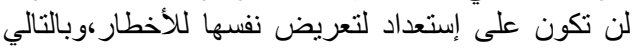

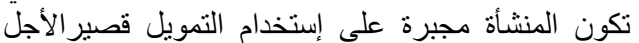

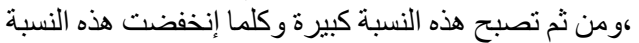

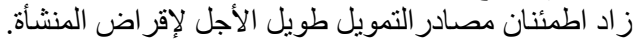

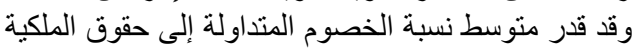

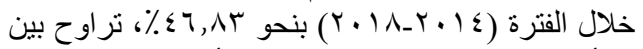

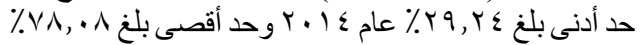

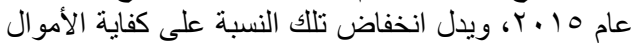

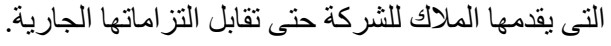

نسبة الإقتر اض إلى رأسال مال المستثمر:يتم حسابه بقسمة

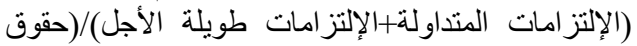

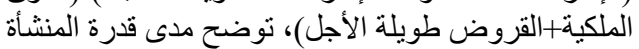

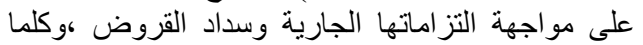

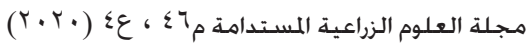




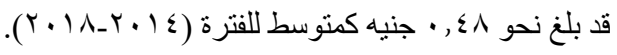

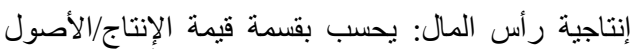

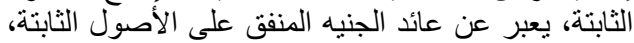

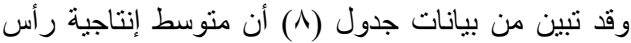

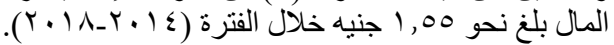

إنتاجية جنيه الأجور: يحسب بقسمة قيمة الإنتاج/قيمة الأجور، وهى تعبر عن عائد الجنيه المنفق على الإلى العمالة،

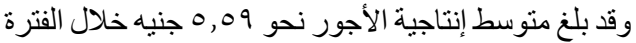

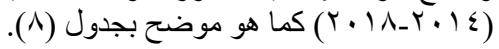

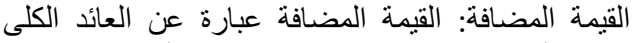

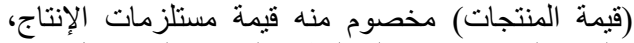

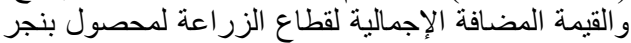

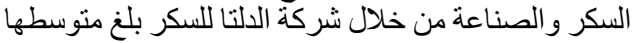

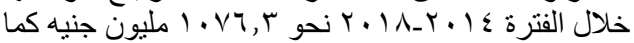

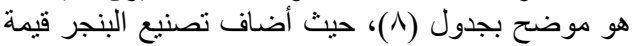

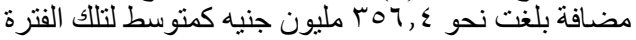
من خلال تصنيعه بشركة الدلتا للسكر.

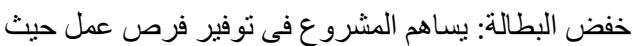

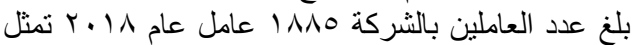

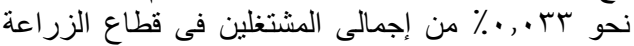

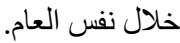

العائد على ر أس المال المستثمر : يتم حسابه بقسمة الأرباح

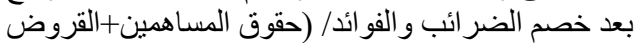
طويلة الأجل)، وقد قدر منوسط العائد على رالس الس المال

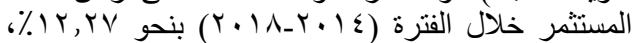

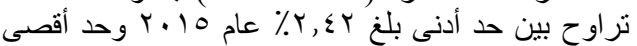

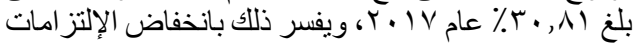

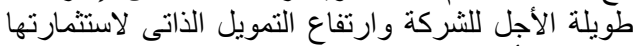
وارتفاع الأرباح.

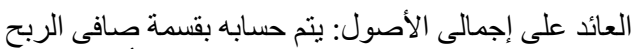

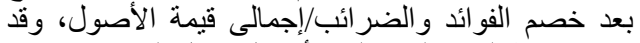

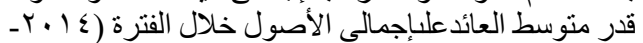

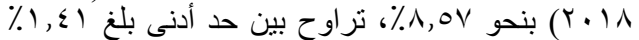

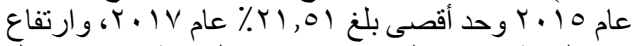

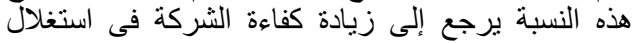

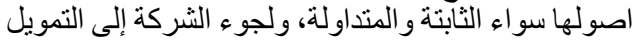

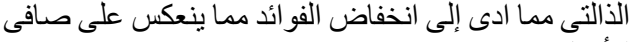

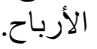

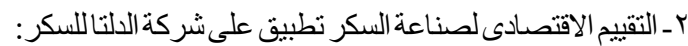

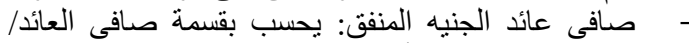

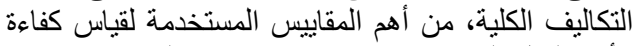

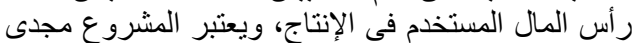

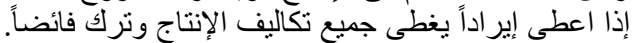
ويشير جدول (^) إلى أن متوسط حسافى عائد الجنيه المنفق

\begin{tabular}{|c|c|c|c|c|c|c|}
\hline المتوسط & $r \cdot 11$ & $r \cdot i v$ & 5.17 & $r+10$ & $r \cdot 1 \leq$ & 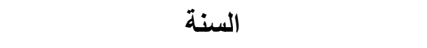 \\
\hline $1, \leqslant V$ & 1,01 & $1,7 V$ & $1, r q$ & $1, .7$ & $1, v \leqslant$ & 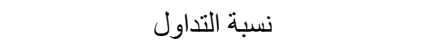 \\
\hline$\cdot$, Or & $\cdot, \leqslant r$ & $\cdot, \leqslant 0$ & $\cdot, V T$ & $\cdot$, to & $\cdot, V T$ & نسبة السيولة \\
\hline $17, \leqslant \wedge$ & $0, \varepsilon r$ & $17,1 \mathrm{~V}$ & $10, \cdot v$ & 10,94 & $(9, \wedge)$ & معدل تغطية الأصول الثابتة \\
\hline$\varepsilon, r q *$ & $\vee, 91$ & $r, r \varepsilon$ & $r, 99$ & $0, \vee 9$ & $r, \varepsilon r$ & نسبة الفو ائد إلى المبيعات٪ \\
\hline$\cdot, \wedge 9$ & $1, \ldots$ & $\cdot, 94$ & 1,11 & $\cdot, 09$ & $\cdot, 10$ & معدل دور ان إجمالى الأصول \\
\hline $1, T V$ & $r, r)$ & 1,91 & 1,74 & $1, \cdot 1$ & $1, r \wedge$ & معدل دوران الأصول الثابتة \\
\hline$r, \cdot V$ & 1,10 & $1, V V$ & $r, r$ & $1, r_{1}$ & $r, r T$ & معدل دوران الأصول المتداولة \\
\hline 1, ro & 1,71 & $1, r v$ & 1,00 & $1, \cdot 1$ & $1,1 \pi$ & معدل دور مان حقوق الملكية \\
\hline $1, r$ & 1,07 & 1, & $1, \leqslant 9$ & $1, \cdot \varepsilon$ & $1, \cdot 9$ & معدل دوران رأس المال المستثمر \\
\hline$\lambda r, O V *$ & $79,0 \mathrm{~V}$ & V1, qr & $94, \varepsilon$ & $99, \wedge$ & $\Lambda T, Y V$ & نسبة الأصول الثابتة إلى حقوق الملكية/\% \\
\hline$\leqslant \neg, \wedge{ }^{*} *$ & $0 \wedge, Y_{T}$ & $\leqslant 7$, or & $r ч, r q$ & $\vee \wedge, \cdot \wedge$ & $r q, r \leq$ & نسبة الخصوم المتداولة إلى حقوق الملكية٪\% \\
\hline$\{\vee, 77 *$ & $0 \leqslant, r \wedge$ & $\sum Y, O r$ & $r \Lambda, \cdot r$ & $V V, T \leq$ & TY,YT & نسبة الإقتر اض إلى رأس المال المستثر ٪ \\
\hline$r, v o *$ & $\vee, 9 \leq$ & r, १४ & $\{, \varepsilon 1$ & $r, \varepsilon r$ & $r, \cdot v$ & نسبة الإقتر اض إلى إجمالى الأصول٪ \\
\hline $0, V Y *$ & $|r, \wedge|$ & $\varepsilon, \leqslant 0$ & $7, r$ & $T, r V$ & $r, V \uparrow$ & نسبة الإقتر اض إلى حقوق الملكية\% \\
\hline $11,1 \vee *$ & שr, & $r q, \wedge$ & $\vee, \wedge \vee$ & r, ro & $19, \varepsilon$ & 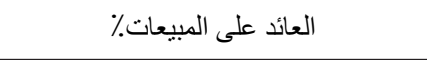 \\
\hline $15, .7 *$ & $r r, Y A$ & $r, \mid \Lambda$ & $|r, r|$ & $r, O V$ & $17,0 r$ & العائد على حقوق الملكية.\% \\
\hline$I r, r V *$ & $r \cdot, r$ & $r \cdot, \lambda)$ & 11,0 & $r, \varepsilon r$ & $17, \cdot 1$ & العائد على رأس المال المستثمر \% \\
\hline$\wedge, \circ \vee *$ & $1 \varepsilon, 1 Y$ & $r 1,01$ & $\wedge, \vee$ & $1, \varepsilon 1$ & $|r, \Sigma|$ & العائد على إجمالى الأصول٪ \\
\hline
\end{tabular}




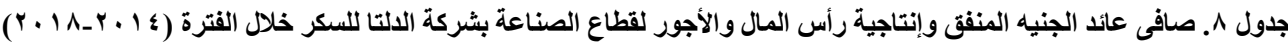

\begin{tabular}{|c|c|c|c|c|c|c|}
\hline مليون جنيه المضافة القيمة & للتيمة المضافة التيع & لزئرة القة البنجر & جنيه الأجور & رأس المالية & الجنيه المنفق عائد & السنة \\
\hline$\cdots r, r$ & rvq,. & VTT,Y & 0,1 & $1, V Y$ & $\cdot, 91$ & r. Is \\
\hline$V \leqslant r, r$ & $V Y$, & $T T V, r$ & $0,1 r$ & $1, r \wedge$ & $\cdot, 0$ & $r .10$ \\
\hline Tor, $\mathrm{V}$ & lor,r & $0 \ldots, 0$ & 7,01 & $1, \cdot 1$ & $\cdot, \cdot r$ & $r .17$ \\
\hline ITrT,O & $V V \cdot, 1$ & Nor, \{ & $0, \pi r$ & 1,77 & $\cdot, r$. & r. IV \\
\hline |זו,. & $0 . \varepsilon, \lambda$ & $107, \mathrm{r}$ & $0, \wedge \wedge$ & 1,91 & $\cdot, 70$ & $r \cdot 11$ \\
\hline $1 \cdot V 7, r$ & rot, ₹ & $v 19,9$ & 0,09 & 1,00 & $\cdot, \leqslant \wedge$ & المتوسط \\
\hline
\end{tabular}

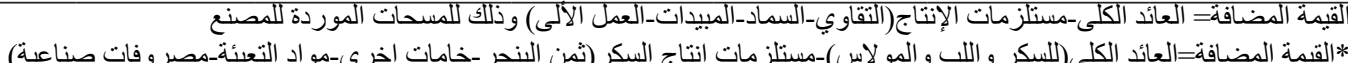

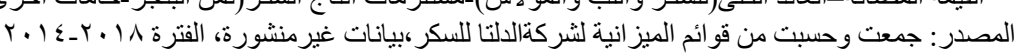

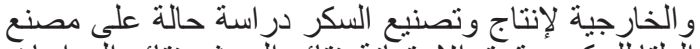

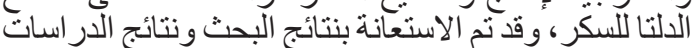

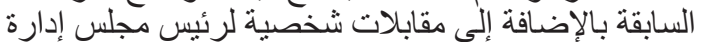

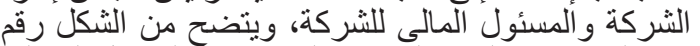

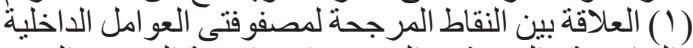

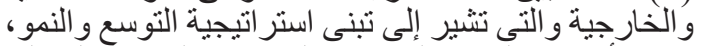

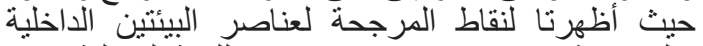

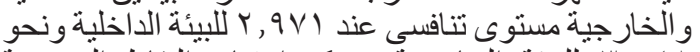

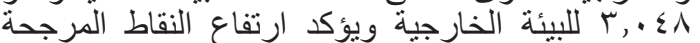

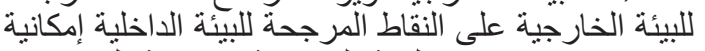

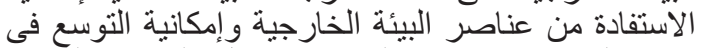

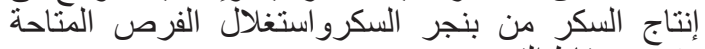
وُتعزيز نقاط القوة.

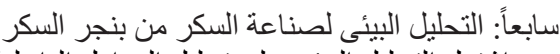

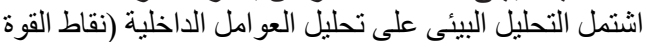

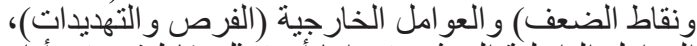
العوامل الداخلية المؤثرة في إما أن تمثل نقاط فوأن فوة في أداء

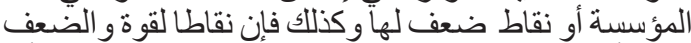

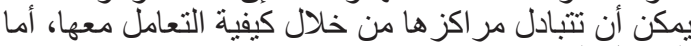

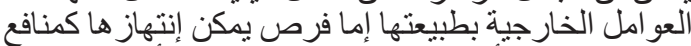

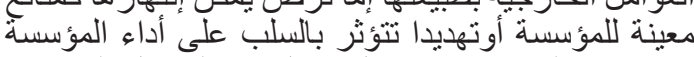

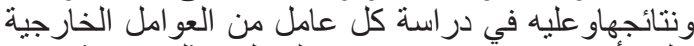

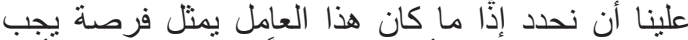

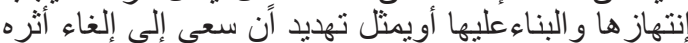

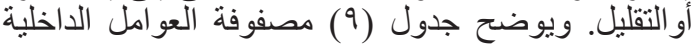

جدول 9.

\begin{tabular}{|c|c|c|c|c|c|c|c|}
\hline \multicolumn{4}{|c|}{ مصفوفة العوامل الخارجية } & \multicolumn{4}{|c|}{ مصفوفة العوامل الداخلية } \\
\hline 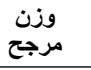 & ترتيب & 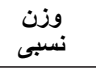 & 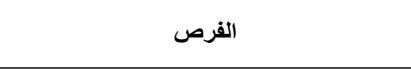 & 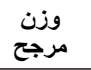 & ترتيب & 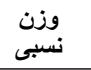 & نقاط القوة \\
\hline$\cdot, \varepsilon 1 \wedge$ & $\varepsilon$ & $\cdot, 1.0$ & السكع الخيمة المضتوردة للصناعة بزيادة تكرير & $\cdot, \varepsilon r\}$ & $\varepsilon$ & $\cdot, 1 \cdot 7$ & زيادة مساهمة إنتاج السكر من البنجر \\
\hline$\cdot, \leq \cdot 7$ & $\varepsilon$ & $\cdot, 1 \cdot 1$ & تجودة زراعة بنجر السكر فى أراضى قليلة & $\cdot, \varepsilon Y V$ & $\varepsilon$ & $\cdot, 1 \cdot v$ & 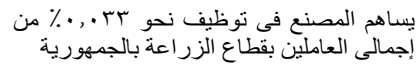 \\
\hline$\cdot, \varepsilon 1 \wedge$ & $\varepsilon$ & $\cdot 1.0$ & أهتمام استرول اتيجية وزارة الزراعة بزيادة & $\cdot, \varepsilon r r$ & $\varepsilon$ & $\cdot 1 \cdot 7$ & المبيعات الإتخدام الكفء لر أس المال المستثر فى توليد \\
\hline$\cdot, \pi)$ & $r$ & $\cdot, 1 \cdot \varepsilon$ & 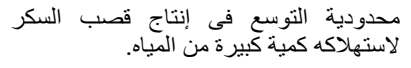 & $\cdot, \varepsilon \cdot 1$ & $\varepsilon$ & $\cdot, 1 \ldots$ & 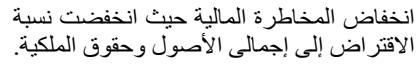 \\
\hline$\cdot, \varepsilon)$ & $\varepsilon$ & $\cdot, 1 \cdot r$ & امكانية استتباط اصناف جديدة انتاجيتها عالية & $\cdot, \mathrm{MIV}$ & r & $\cdot, 1 \cdot 7$ & نجاح الأداء المالى للشركة. \\
\hline$\cdot$, rqV & $\varepsilon$ & $\cdot, .99$ & التخطيط لإنشاء مصانع جديدة & $\cdot, \Gamma) \leq$ & r & $\cdot, 1.0$ & أى قيمة مضافة من المنتجات الثانوية لتصنيع البنجر \\
\hline 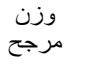 & ترتيب & 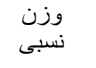 & التهديدات & 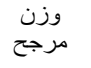 & ترنيب & 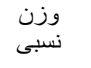 & 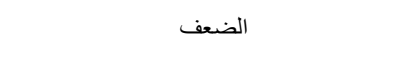 \\
\hline$\cdot,|v|$ & r & $\cdot, \cdot 10$ & انخفاص الأسعار العالمية للسكر & $\cdot, I V Y$ & r & $\cdot, \cdot 14$ & وجود فجوة بين الإنتاج و الاستهلاك من السكر. \\
\hline$\cdot, 17$ & r & $\cdot, \wedge$ & السكر. أسعار الزروع الثتوية المنافسة لبنجر &., $1 Y 9$ & r & $\cdot,+70$ & انخفاض إنتاجية الفدان فى بعض المحافظات \\
\hline$\cdot, 1 \leq 9$ & r & $\cdot, \cdot v_{0}$ & ارتفاع أسعار تقاوى البنجر المستوردة. & $\cdot, 1 \times 9$ & r & $\cdot, \cdot 70$ & ارتفاع تكلفة إنتاج الفدان من بنجر السكر. \\
\hline$\cdot, \mid r \wedge$ & r & $\cdot, \cdot 7 \leqslant$ & 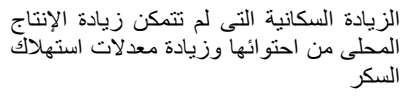 & $\cdot, 17 r$ & r & $\cdot, \cdot 11$ & 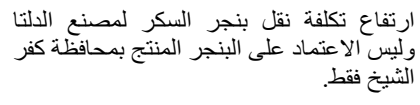 \\
\hline$\cdot, \cdot \wedge$ & 1 & $\cdot, \wedge$. & انخفاض أسعار توريد البنجر & $\cdot, \cdot$ vo & 1 & $\cdot, \cdot$ vo & للمدانع. الاستفادة القصوى من الطاقة التصميمية \\
\hline$r, \cdot \leq \wedge$ & $1, \cdot$ & q, rV. & مجموع العو امل الخارجية & r,qV। & $1, \cdot$ & $9, r \wedge$. & مجموع العو امل الداخلية م \\
\hline
\end{tabular}




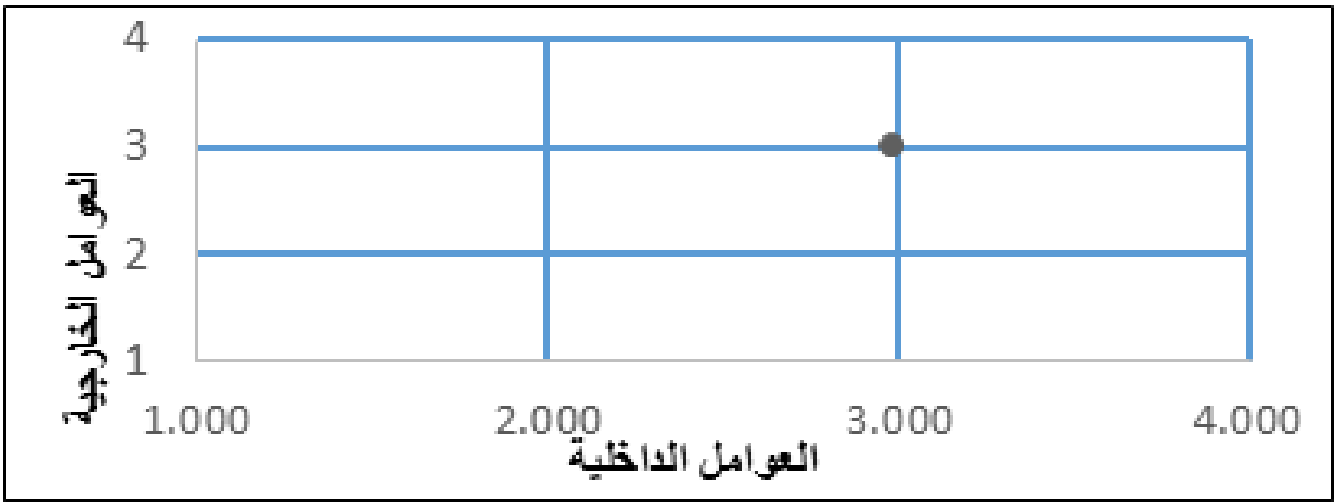

شكل 1. العلاقة بين النقاط المرجحة لمصفوفتى العوامل الاخلية والخارجية

- - العدوى، رشدى شوقى، (Y (Y) دراسة اقتصادية تحليلية

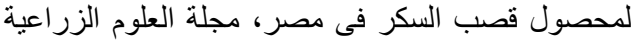

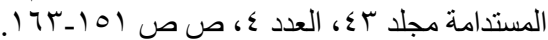

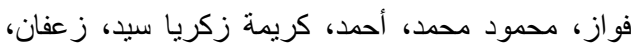

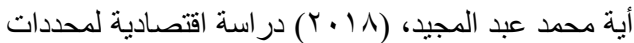

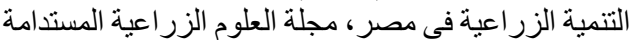

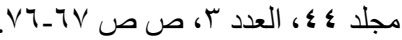

وزارة الزراعة واستصلاح الاراضى،استراتيجية وزارة

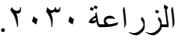

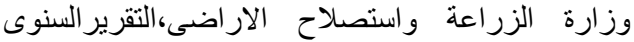

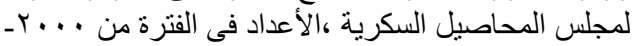
$r \cdot 11$

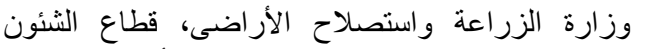

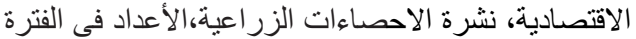

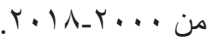

- Bowen, D. J., Kreuter, M., Spring, B., CoftaWoerpel, L., Linnan, L., Weiner, D., \& Fernandez, M. (2009) How we design feasibility studies. American journal of preventive medicine, 36(5), p. 452-457.

- Heinbockel, J. H. (2012) Introduction to calculus. Old Dominion University, p. 388.
وفى ضوء النتائج البحثية ونتائج التحليل البيئى ،فإن الدراسة

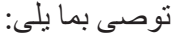

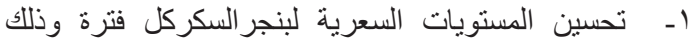

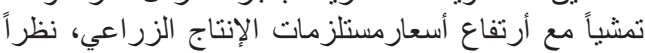

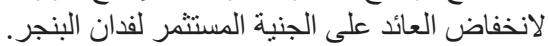

r- - تشغيل مصانع إنتاج السكر بكامل طاقتها بناءاً على زيادة الطاقات الإنتاجية لوجود بعض الطاقات معطلة.

rـ السعى إلى رفع إنتاجية الفدان من بنجر السكر تدريجياً للوصول

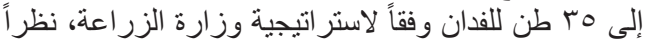

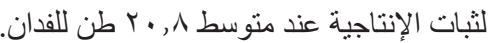

ع- محاولة تخفيض تكاليف انتاج السكر المحلى لوجود تهديد انخفاض الأسعار العالمية للسكر.

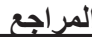

- - الجزار، محمد حمودة، عبد الله، حمزة حامد، محمد، أسماء

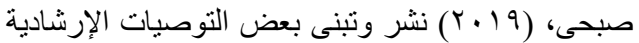
بين زراع محصول بنجر السكر بمركز بيلا محافظة كفر العز

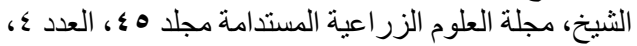

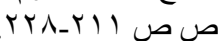
الجهاز المركزى للتعبئة العامة و الإحصاء، الكتاب السنوى،

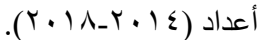

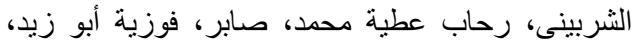

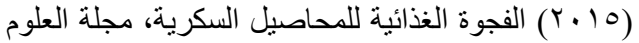

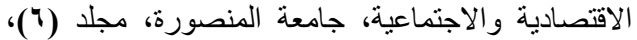

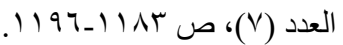




\section{Economic and Environmental Assessment of Sugar Beet Production and Processing in Egypt}

Seham A. A. Hashim

Environmental Agric. Sci. Dept., Institute of Environmental Studies and Research, Ain

Shams Univ., Cairo, Egypt.

$\mathbf{T}$

THE 2030 sustainable agricultural development strategy aimed to increase the produced amount of sugar to about 3.5 million tons by increasing the area planted with sugar beet to reach 800 thousand feddans by 2030 to cover the sugar gap, so the research aims to conduct an economic and environmental assessment of sugar production from sugar beet In Egypt. An evaluation of the financial performance of the Delta Sugar Company for the period (2014-2018) was conducted which showed an increase in the company's ability to fulfill its short-term financial obligations, Fixed assets cover long-term loans, high annual average fixed assets turnover, the company's efficiency in using current assets in the production process and generating sales from them. The company's economic evaluation also revealed that the average net return of the pound amounted to about 0.48 pounds, and the average capital productivity was about 1.55 pounds, and the average productivity of wages was about 5.59 pounds as an average for the period, and the added value of beet processing was about 356.4 million pounds. Through SWOT analysis, the weighted points for the elements of the internal and external environments showed a competitive level at 2,971 for the internal environment and about 3.048 for the external environment. The higher weighted points for the external environment over the weighted points for the internal environment confirm the possibility of expanding sugar production from sugar beet.

Keywords: Sugar beet, Sugar Self-sufficiency, Financial performance, Economic Evaluation, Delta Sugar Factory, Panel data model, SOWT analysis 